\title{
On stochastic modeling of groundwater flow and solute transport in multi-scale heterogeneous formations
}

\author{
BILL X. HU ${ }^{1,2}$, JICHUN WU ${ }^{1,3}$ and CHANGMING HE ${ }^{1,2}$ \\ ${ }^{1}$ Florida State University, Department of Geological Sciences, Tallahassee, FL 32306, USA \\ ${ }^{2}$ Hydrology Program, University of Nevada, Reno, USA \\ ${ }^{3}$ Department of Earth Sciences, Nanjing University, China \\ E-mail: hu@gly.fsu.edu / jcwu@nju.edu.cn / changming.he@dri.edu
}

\begin{abstract}
A numerical moment method (NMM) is applied to study groundwater flow and solute transport in a multiple-scale heterogeneous formation. The formation is composed of various materials and conductivity distribution within each material is heterogeneous. The distribution of materials in the study domain is characterized by an indicator function and the conductivity field within each material is assumed to be statistically stationary. Based on this assumption, a general expression is derived for the covariance function of the composite field in terms of the covariance of the indicator variables and the statistical properties of the composite materials. The NMM is used to investigate the effects of various uncertain parameters on flow and transport predictions in two case studies. It is shown from the study results that the two-scale stochastic processes of heterogeneity will both significantly influence the flow and transport predictions, especially for the variances of hydraulic head and solute fluxes. This study also shows that the NMM can be used to study flow and transport in complex subsurface environments. Therefore, the method may be applicable to complex environmental projects.
\end{abstract}

Mathematical subject classification: $60 \mathrm{H} 30,60 \mathrm{G} 60$.

Key words: heterogeneity, multi-scale, groundwater flow, solute transport.

\section{Introduction}

It is a well known that at a field scale, geological formations are heterogeneous, and the groundwater flow and solute transport processes in the formation are \#570/03. Received: 24/IX/03. Accepted: 29/IV/04. 
considerably affected by the heterogeneity of the formation properties. In the last two decades, many stochastic theories have been developed for groundwater flow and solute transport in heterogeneous porous media [e.g., Dagan, 1989; Gelhar, 1993; Cushman, 1997; Zhang, 2002]. In development of the theories, it is common to assume that the spatial distributions of the medium properties can be characterized by one single correlation scale. This assumption was based on some field studies [Hoeksema and Kitanitis, 1984; Gelhar, 1993], as well as on the notion of the existence of a discrete hierarchy of scales of heterogeneity [Dagan, 1986], with disparity between the scales such that when modeling groundwater flow and solute transport at one scale, variations at other scales can either be averaged out (if other scales are much smaller), or be modeled as a deterministic trend (if other scales are much larger). However, hydraulic properties of many natural media exhibit heterogeneity at multi-scales [Wheatcraft and Tyler, 1998], where the heterogeneity at any scale cannot be averaged out, nor be treated as a deterministic trend.

The usual approach to dealing with multi-scale heterogeneity is simply to increase the variance of stationary processes and not account for the structure of the heterogeneity. This simplification leads to the increase of the variances of hydraulic parameters like conductivity and make the stochastic solution invalid since stochastic groundwater flow models usually rely on small $\sigma_{f}^{2}$, the variance of log transformed conductivity.

In many applications, a random field may be composed of a number of subfields. For example, a geologic formation may consist of several distinct geological units (e.g., layers), each of which may be resulted from different geological processes. Although the permeability of each geologic unit (or subunit) may be a stationary process (field), it has different statistical moments than do its neighboring units. Therefore, the permeability of the entire formation is not a stationary field although that of each subunit is [Zhang, 2002]. In some cases, the large-scale features, such as the locations of geological layers (or zones), can be identified from measurements, only the spatial variability of medium properties, such as the hydraulic conductivity, within each layer (or zone) need to be treated as random processes. Solute transport in this kind of medium has been studied [Zhang et al., 2000; Wu et al., 2003]. But in other cases, the geometries or 
boundaries of the large-scale features cannot be very well defined due to incomplete knowledge about them, they also need to be treated as random variables. This kind of media are called multi-(or dual-) scale heterogeneous media. This study focuses on the influence of the multi-scale heterogeneity on groundwater flow and solute transport processes.

A medium with multi-scale heterogeneity is generally nonstationary (statistically non-omogeneous). Solute transport in a nonstationary permeability field is a relatively new research area. Recently, a theoretical framework for solute flux through spatially nonstationary flows in porous media has been developed [Zhang et al., 2000; Wu et al., 2003]. The solute flux depends on the solute travel time and transverse displacement at a fixed control plane. The solute flux statistics (mean and variance) were derived using a Lagrangian framework and were expressed in terms of the probability density functions (PDFs) of particle travel time and transverse displacement. These PDFs were evaluated through the first two moments of travel time and transverse displacement and the assumed distribution function. This approach was applied to study solute transport in multiscale media, where random heterogeneities exist at some small scale while deterministic geological structures and patterns can be prescribed at some larger scale. Lu and Zhang [2002] applied moment equation approach to study groundwater flow in a multimodal heterogeneous medium, where the large-scale heterogeneity, the distribution of the various media, is also stationary. In this study, we will firstly extend the flow study to a multi-scale medium, then study solute transport in this medium and also in a multimodal medium.

\section{Mean and covariance of a log-hydraulic conductivity field in a multiple-scale heterogeneous formation}

Let us consider a formation composed of $k$ distinct features (e.g., layers, zones, or facies). The process of $\log$ hydraulic conductivity, $Y(\mathbf{x})$ of the formation is represented by a $k$-dimensional stochastic process, $Z(\mathbf{x})=\left(Y_{1}(\mathbf{x}), \Lambda, Y_{k}(\mathbf{x})^{\prime}\right.$, and the indicator, $I(\mathbf{x})$, is represented by a process $I(\mathbf{x})=\left(I_{1}(\mathbf{x}), \Lambda, I_{k}(\mathbf{x})\right)^{\prime}$, where the possible values of $I(\mathbf{x})$ are $R^{k}$ base vectors: $e_{1} . e_{2}, \Lambda, e_{k}$, that is each of $e / s$ is a $k$-dimensional vector with an 1 on $i$ 'th coordinate and 0 on the rest of the coordinates. The formulation in terms of the base vectors is easier for 
the definition of the final process $Y(\mathbf{x})$ as a dot product (below), which is the only reason we used it. Further, the probability that $I(\mathbf{x})=e_{1}$ is $p_{1}(x)$, that is $p\left(I(\mathbf{x})=e_{1}\right)=p_{1}(\mathbf{x})$ for $i=1, \ldots, k$. The relationship between the two stochastic processes are assumed to be mutual independent, which means that $I(\mathbf{x})$ is independent (as a process) from $Z(\mathbf{x})$, and vice versa. The process of $Y(\mathbf{x})$ is defined as,

$$
Y(\mathbf{x})=\sum_{i=1}^{k} Y_{i}(\mathbf{x}) I_{i}(\mathbf{x})
$$

$Y(\mathbf{x})$ is a scalar product. This definition of $Y(\mathbf{x})$ generalizes the processes described by Rubin [1995] and Zhang [2002], where $k=2$.

Under the condition of mutual independence between $I$ and $Z$, we obtain the mean of $Y$ in a two-scale heterogeneous formation as

$$
\langle Y(\mathbf{x})\rangle=\sum_{i=1}^{k}\left\langle Y_{i}(\mathbf{x})\right\rangle p_{i}(\mathbf{x})
$$

By definition, the covariance of $Y$ at any two points, $\mathbf{x}$ and $\mathbf{s}$, are expressed as

$$
\begin{aligned}
C_{Y}(\mathbf{x}, \mathbf{s}) & =\langle[Y(\mathbf{x})-\langle Y(\mathbf{x})\rangle][Y(\mathbf{x})-\langle Y(\mathbf{x})\rangle]\rangle \\
& =\langle Y(\mathbf{x}) Y(\mathbf{s})\rangle-\langle Y(\mathbf{x})\rangle\langle Y(\mathbf{x})\rangle
\end{aligned}
$$

Based on (2), $\langle Y(\mathbf{x})\rangle\langle Y(\mathbf{s})\rangle$ can be derived as

$$
\begin{aligned}
\langle Y(\mathbf{x})\rangle\langle Y(\mathbf{x})\rangle & =\sum_{i=1}^{k}\left\langle Y_{i}(\mathbf{x})\right\rangle p_{i}(\mathbf{x}) \sum_{j=1}^{k}\left\langle Y_{j}(\mathbf{x})\right\rangle p_{j}(\mathbf{s}) \\
& =\sum_{i, j=1}^{k}\left\langle Y_{i}(\mathbf{x})\right\rangle\left\langle Y_{j}(\mathbf{s})\right\rangle p_{i}(\mathbf{x}) p_{j}(\mathbf{s})
\end{aligned}
$$

We define two notations,

$$
\begin{aligned}
& p_{i j}(\mathbf{x}, \mathbf{s})=p\left(I(\mathbf{x})=e_{i}, I(\mathbf{s})=e_{j}\right) \quad \text { and } \\
& C_{Y, i j}(\mathbf{x}, \mathbf{s})=\left\langle\left[Y_{i}(\mathbf{x})-\left\langle Y_{i}(\mathbf{x})\right\rangle\right]\left[Y_{j}(\mathbf{s})-\left\langle Y_{j}(\mathbf{s})\right\rangle\right]\right\rangle .
\end{aligned}
$$


Then, $\langle Y(\mathbf{x}) Y(\mathbf{s})\rangle$ is derived as,

$$
\begin{aligned}
\langle Y(\mathbf{x}) Y(\mathbf{s})\rangle & =\sum_{i, j=1}^{k}\left\langle Y_{i}(\mathbf{x}) Y_{j}(\mathbf{s}) \mid I(\mathbf{x})=e_{i}, I(\mathbf{s})=e_{j}\right\rangle p_{i j}(\mathbf{x}, \mathbf{s}) \\
& =\sum_{i \neq j}\left\langle Y_{i}(\mathbf{x}) Y_{j}(\mathbf{s})\right\rangle p_{i j}(\mathbf{x}, \mathbf{s})+\sum_{i=1}^{k}\left\langle Y_{i}(\mathbf{x}) Y_{i}(\mathbf{s})\right\rangle p_{i i}(\mathbf{x}, \mathbf{s})
\end{aligned}
$$

In the derivation of (5), independence of $Y$ and $I$ is applied. Further, due to the independence of $Y_{i}^{\prime}$ and $Y_{j}^{\prime}$, where $Y_{l}^{\prime}=Y_{l}-\left\langle Y_{l}\right\rangle(l=i, j)$, we can obtain

$$
\begin{aligned}
\langle Y(\mathbf{x}) Y(\mathbf{s})\rangle= & \sum_{i \neq j}\left\langle Y_{i}(\mathbf{x})\right\rangle\left\langle Y_{j}(\mathbf{s})\right\rangle p_{i j}(\mathbf{x}, \mathbf{s}) \\
& +\sum_{i=1}^{k}\left\{C_{Y, i i}(\mathbf{x}, \mathbf{s})+\left\langle Y_{i}(\mathbf{x})\right\rangle\left\langle Y_{i}(\mathbf{s})\right\rangle\right\} p_{i i}(\mathbf{x}, \mathbf{s}) \\
= & \sum_{i, j=1}^{k}\left\langle Y_{i}(\mathbf{x})\right\rangle\left\langle Y_{j}(\mathbf{s})\right\rangle p_{i j}(\mathbf{x}, \mathbf{s})+\sum_{i=1}^{k} C_{Y, i i}(\mathbf{x}, \mathbf{s}) p_{i i}(\mathbf{x}, \mathbf{s})
\end{aligned}
$$

From (4) and (6), the expression of $C_{Y}(\mathbf{x}, \mathbf{s})$ is obtained as

$$
\begin{aligned}
C_{Y}(\mathbf{x}, \mathbf{s})= & \sum_{i, j=1}^{k}\left\langle Y_{i}(\mathbf{x})\right\rangle\left\langle Y_{j}(\mathbf{s})\right\rangle p_{i j}(\mathbf{x}, \mathbf{s})-p_{i}(\mathbf{x}) p_{j}(\mathbf{s}) \\
& +\sum_{i=1}^{k} C_{Y, i i}(\mathbf{x}, \mathbf{s}) p_{i i}(\mathbf{x}, \mathbf{s})
\end{aligned}
$$

Equation (7) is the general expression of $C_{Y}(\mathbf{x}, \mathbf{s})$ without any assumption on the structure of the process $I$. For a specific field site, we need to obtain the joint probability, $p_{i j}(\mathbf{x}, \mathbf{s})$, from field investigations to calculate $C_{Y}(\mathbf{x}, \mathbf{s})$.

The correlation structure of the process $I$ can be expressed as $C_{I, i j}(\mathbf{x}, \mathbf{s})=$ $p_{i j}(\mathbf{x}, \mathbf{s})-p_{i}(\mathbf{x}) p_{j}(\mathbf{s})$. Equation (7) can be rewritten as

$$
\begin{aligned}
C_{Y}(\mathbf{x}, \mathbf{s})= & \sum_{i, j=1}^{k}\left\langle Y_{i}(\mathbf{x})\right\rangle\left\langle Y_{j}(\mathbf{s})\right\rangle C_{I, i j}(\mathbf{x}, \mathbf{s}) \\
& +\sum_{i=1}^{k} C_{Y, i i}(\mathbf{x}, \mathbf{s})\left(C_{I, i i}(\mathbf{x}, \mathbf{s})+p_{i}(\mathbf{x}) p_{i}(\mathbf{s})\right)
\end{aligned}
$$


Equation (8) is the expression for the covariance function of the composite field in terms of the covariance of the indicator variables and the properties of the composite materials.

The expressions of conductivity covariance in two-scale heterogeneous media in (7) and (8) are equivalent to those derived by Lu and Zhang [2002] and generalize the previous studies on many specific cases [e.g., Zhang 2002]. By setting $\mathbf{x}=\mathbf{s}$ in (7), or (8), the variance of $Y(\mathbf{x})$ is obtained as

$$
\begin{aligned}
\sigma_{Y}^{2}(\mathbf{x})= & \sum_{i=1}^{k} p_{i}(\mathbf{x}) \sigma_{Y_{i}}^{2}(\mathbf{x})+\sum_{i=1}^{k} p_{i}(\mathbf{x})\left\langle Y_{i}(x)\right\rangle^{2} \\
& -\sum_{i, j=1}^{k} p_{i}(\mathbf{x}) p_{j}(\mathbf{x})\left\langle Y_{i}(\mathbf{x})\right\rangle\left\langle Y_{j}(\mathbf{x})\right\rangle
\end{aligned}
$$

It is obvious that the distribution of $Y(\mathbf{x})$ can be highly nonstationary even though the distribution of $Y_{i}(\mathbf{x})$ in each subunit is stationary. If the two stochastic processes of $I(\mathbf{x})$ and $Y_{i}(\mathbf{x})$ are both stationary, then $\sigma_{Y}^{2}$ will be a constant.

\section{The statistics of hydraulic conductivity in special cases}

The general expression of the velocity covariance in a two-scale heterogeneous medium is very complicated. The expression can be greatly simplified in some specific cases. Here we will use couple of examples to show to how to simplify the results according to the specific field conditions. A few studies have been conducted to study a natural medium with bimodal distribution of hydraulic conductivity [e.g., Desbarats, 1987, 1991; Rubin and Journel, 1991; Russo et al., 2001]. The bimodal distribution is a special case of this study. Let $k=2$ in (8), we obtain the covariance of log-hydraulic conductivity in a bimodal heterogeneous medium as,

$$
\begin{aligned}
C_{Y}(\mathbf{x . s})= & \left\langle Y_{1}(\mathbf{x})\right\rangle\left\langle Y_{1}(\mathbf{s})\right\rangle C_{I, 11}(\mathbf{x}, \mathbf{s})+\left\langle Y_{2}(\mathbf{x})\right\rangle\left\langle Y_{1}(\mathbf{s})\right\rangle C_{I, 22}(\mathbf{x}, \mathbf{s}) \\
& +\left\langle Y_{1}(\mathbf{x})\right\rangle\left\langle Y_{2}(\mathbf{s})\right\rangle C_{I, 12}(\mathbf{x}, \mathbf{s})+\left\langle Y_{2}(\mathbf{x})\right\rangle\left\langle Y_{1}(\mathbf{s})\right\rangle C_{I, 21}(\mathbf{x}, \mathbf{s}) \\
& +C_{Y, 11}(\mathbf{x}, \mathbf{s})\left(C_{I, 11}(\mathbf{x}, \mathbf{s})+p_{1}(\mathbf{x}) p_{1}(\mathbf{x})\right) \\
& +C_{Y, 22}(\mathbf{x}, \mathbf{s})\left(C_{I, 22}(\mathbf{x}, \mathbf{s})+p_{2}(\mathbf{x}) p_{2}(\mathbf{s})\right)
\end{aligned}
$$


Using the relationships (the derivation is shown in Appendix)

$$
C_{I, 11}+C_{I, 12}=0, C_{I, 21}+C_{I, 22}=0
$$

(10) can be rewritten as

$$
\begin{aligned}
C_{Y}(\mathbf{x}, \mathbf{s})= & C_{Y, 11}(\mathbf{x}, \mathbf{s})\left(C_{I, 11}(\mathbf{x}, \mathbf{s})+p_{1}(\mathbf{x}) p_{1}(\mathbf{s})\right) \\
& +C_{Y, 22}(\mathbf{x}, \mathbf{s})\left(C_{I, 22}(\mathbf{x}, \mathbf{s})+p_{2}(\mathbf{x}) p_{2}(\mathbf{s})\right) \\
& +\left\langle Y_{1}(\mathbf{x})\right\rangle\left\langle Y_{1}(\mathbf{s})\right\rangle C_{I, 11}(\mathbf{x}, \mathbf{s})+\left\langle Y_{2}(\mathbf{x})\right\rangle\left\langle Y_{2}(\mathbf{s})\right\rangle C_{I, 22}(\mathbf{x}, \mathbf{s}) \\
& -\left\langle Y_{1}(\mathbf{x})\right\rangle\left\langle Y_{2}(\mathbf{s})\right\rangle C_{I, 11}(\mathbf{x}, \mathbf{s})-\left\langle Y_{2}(\mathbf{x})\right\rangle\left\langle Y_{1}(\mathbf{s})\right\rangle C_{I, 22}(\mathbf{x}, \mathbf{s})
\end{aligned}
$$

By using the relationships in bimodal medium, $p_{2}(\mathbf{x})=1-p_{1}(\mathbf{x})$ and $p_{2}(\mathbf{s})=$ $1-p_{1}(\mathbf{s})$, and assuming $C_{I, 11}(\mathbf{x}, \mathbf{s})=C_{I, 22}(\mathbf{x}, \mathbf{s})=C_{I}(\mathbf{x}, \mathbf{s})$, then (12) can be simplified as

$$
\begin{aligned}
C_{Y}(\mathbf{x}, \mathbf{s})= & C_{Y, 11}(\mathbf{x}, \mathbf{s})\left[C_{I}(\mathbf{x}, \mathbf{s})+p_{1}(\mathbf{x}) p_{1}(\mathbf{s})\right] \\
& +C_{Y, 22}(\mathbf{x}, \mathbf{s})\left\{C_{I}(\mathbf{x}, \mathbf{s})+\left[1-p_{1}(\mathbf{x})\right]\left[1-p_{1}(\mathbf{s})\right]\right\} \\
& +C_{I}(\mathbf{x}, \mathbf{s})\left[\left\langle Y_{1}(\mathbf{x})\right\rangle-\left\langle Y_{2}(\mathbf{x})\right\rangle\right]\left[\left\langle Y_{1}(\mathbf{s})\right\rangle-\left\langle Y_{2}(\mathbf{s})\right\rangle\right]
\end{aligned}
$$

Equation (13) is the same as the result in Zhang [2002].

Setting $\mathbf{s}=\mathbf{x}$ in (13), we can obtain the variance of $Y(\mathbf{x})$ in the bimodal medium as

$$
\begin{aligned}
\sigma_{Y}^{2}(\mathbf{x})= & p_{1}(\mathbf{x}) \sigma_{1}^{2}(\mathbf{x})+p_{2}(\mathbf{x}) \sigma_{2}^{2}(\mathbf{x})+\left(\left\langle Y_{1}(\mathbf{x})\right\rangle-\left\langle Y_{2}(\mathbf{x})\right\rangle\right)^{2} \\
& \left.-\left\langle Y_{2}(\mathbf{x})\right\rangle\right)^{2} p_{1}(\mathbf{x}) p_{2}(\mathbf{x})=p_{1}(\mathbf{x}) \sigma_{1}^{2}(\mathbf{x})+\left[1-p_{1}(\mathbf{x})\right] \sigma_{2}^{2}(\mathbf{x}) \\
& +\left(\left\langle Y_{1}(\mathbf{x})\right\rangle-\left\langle Y_{2}(\mathbf{x})\right\rangle\right)^{2} p_{1}(\mathbf{x})\left[1-p_{1}(\mathbf{x})\right]
\end{aligned}
$$

If we further assume that $I(x)$ is also stationary as the case considered by Rubin and Journel [1991], then Eqs. (2), (13) and (14) become

$$
\begin{aligned}
\langle Y\rangle= & p_{1}\left\langle Y_{1}\right\rangle+\left(1-p_{1}\right)\left\langle Y_{2}\right\rangle \\
C_{Y}(\mathbf{x}, \mathbf{s})= & C_{Y}(\mathbf{x}-\mathbf{s})=C_{Y}(\mathbf{r}) \\
= & C_{Y, 11}(\mathbf{r})\left[C_{I}(\mathbf{r})+p_{1}^{2}\right]+C_{Y, 22}(\mathbf{r})\left\{C_{I}(\mathbf{r})+\left[1-p_{1}\right]^{2}\right\} \\
& +C_{I}(\mathbf{r})\left[\left\langle Y_{1}\right\rangle-\left\langle Y_{2}\right\rangle\right]^{2} \\
\sigma_{Y}^{2}= & p_{1} \sigma_{1}^{2}+\left(1-p_{1}\right) \sigma_{2}^{2}+\left(\left\langle Y_{1}\right\rangle-\left\langle Y_{2}\right\rangle\right)^{2} p_{1}\left(1-p_{1}\right)
\end{aligned}
$$


From (15)-(17), one may notice that $\langle Y\rangle$ and $\sigma_{Y}^{2}$ are constant, and $C_{Y}(\mathbf{x}, \mathbf{s})$ is stationary. If the difference between the two $\left\langle Y_{1}\right\rangle$ and $\left\langle Y_{2}\right\rangle$ is large, then the third terms in (16) and (17) will be dominant for the covariance and variance, respectively. For this reason, Desbarats [1987] treated $Y_{1}$ and $Y_{2}$ as constants in a sand-shale formation, only $I(\mathbf{x})$ is assumed to be a stochastic process. In this special case, (16)-(17) can be further simplified as

$$
\begin{aligned}
C_{Y}(\mathbf{r}) & =C_{I}(r)\left[\left\langle Y_{1}\right\rangle-\left\langle Y_{2}\right\rangle\right]^{2} \\
\sigma_{Y}^{2} & =\left(\left\langle Y_{1}\right\rangle-\left\langle Y_{2}\right\rangle\right)^{2} p_{1}\left(1-p_{1}\right)
\end{aligned}
$$

Therefore, we conclude that the expression of the conductivity covariance developed in this study generalizes previous study results. The general expression of the covariance in a multi-scale medium may be significantly simplified in special cases.

\section{Method of moment for groundwater glow and solute transport in nonstationary heterogeneous media}

In the last two sections, the explicit expressions for the mean, variance and covariance of hydraulic conductivity in a dual-scale medium have been given in terms of the means, variances of the indicator variable and the hydraulic conductivity of the every single material in the medium, which can be obtained through field measurements and geostatistical analysis. The obtained statistics of the hydraulic conductivity will be used as the input data for the calculations of flow and transport.

Zhang and Winter [1999] developed a numerical moment approach for groundwater flow in a stationary conductivity field in a bounded domain. Later, Lu and Zhang [2002] extended the method to study groundwater flow in a multi-scale medium. In their study, they also conducted Monte Carlo simulation, and the study results indicate that the method of moment is consistent with Monte Carlo simulation even for the total variance of log-conductivity is 4.0. In this study we apply the method to study groundwater flow in nonstationary conductivity fields with complex covariance functions and limited boundary conditions. The resulting velocity moments will be served as the input data for the transport cal- 
culation. In this article, the method which is similar to Lu and Zhang's [2002] work will not be presented, but the calculated results for the head and velocity will be shown in our case studies.

Recently, a numerical method of moment for solute transport in a nonstationary flow field has been developed. Zhang et al. [2000] and Wu et al. [2003] applied a perturbation approach to set up an analytical framework for solute transport in a nonstationary flow field. The method is to predict solute flux in a two-dimensional flow field through a control plane (CP), which is located at some distance downstream from the plume source. The mean solute mass flux component orthogonal to the $\mathrm{CP}$ at $x,\langle q(t, \mathbf{x})\rangle$, can be expressed as

$$
\langle q(t, \mathbf{x})\rangle=\int_{A_{0}} \int_{0}^{\infty} \rho_{0}(a) \phi(t-\tau) f_{1}[\tau(x, a)=t, \eta(x, \mathbf{a})=y] d \tau d \mathbf{a}
$$

where $\mathbf{x}=(x, y)$ is the solute predicting point, $\tau \equiv \tau(x ; \mathbf{a})$ is the travel time of the advective particle from point a to the control plane at $x, \eta(\mathbf{x} ; a)$ is the transverse location of a streamline passing through the $\mathrm{CP}, A_{0}$ is the source area, $\rho_{0}(\mathbf{a})$ is the density of the source mass, $\phi(t)$ is the source release function. $f_{1}[\tau(x, a), \eta(x, \mathbf{a})]$ denotes the joint probability density function (PDF) of travel time $\mathrm{t}$ for a particle from $\mathrm{a}$ to reach $x$ and the corresponding transverse displacement $\eta$. The $\tau$ and $\eta$ are random variables and are functions of the underlying random velocity field.

The variance of the solute flux for the point sampling is evaluated as

$$
\sigma_{q}^{2}(t, \mathbf{x})=\left\langle q^{2}\right\rangle-\langle q\rangle^{2}
$$

where

$$
\begin{aligned}
\left\langle q^{2}(t, \mathbf{x})\right\rangle & =\iint_{A_{0}} \int_{A_{0}} \rho_{0}(\mathbf{a}) \rho_{0}(\mathbf{b}) \phi\left(t-\tau_{1}\right) \phi\left(t-\tau_{2}\right) \cdot f_{2}\left[\tau_{1}(x, \mathbf{a})\right. \\
& \left.=t, \eta_{1}(x, \mathbf{a})=y, \tau_{2}(x, \mathbf{b})=t, \eta_{2}(x, \mathbf{b})=y\right] d \mathbf{a} d \mathbf{b}
\end{aligned}
$$

Here $f_{2}\left[\tau_{1}(x, a), \eta_{1}(x, \mathbf{a}) ; \tau_{2}(x, b), \eta_{2}(x, \mathbf{b})\right]$ is the two-particle joint PDF of travel time and transverse displacement. The solute discharge, $Q(t, x)$, defined as the total solute mass flux over the entire control CP, can be obtained by integration $q(t, \mathbf{x})$ along the CP. 
The travel time $\tau(L ; \mathbf{a})$, the time required for a parcel originated at a to cross the plane $x=L$, is determined by the Lagrangian velocity, which is related to the Eulerian velocity. The mean $\langle\tau\rangle$ and fluctuation of $\tau(L ; \mathbf{a}), \tau^{\prime}$, is expressed, to the first order, as

$$
\begin{aligned}
\langle\tau(L ; \mathbf{a})\rangle & =\int_{a_{x}}^{L} \frac{d x}{U_{1}(x,\langle\eta\rangle)} \\
\tau^{\prime}(L ; \mathbf{a}) & =-\int_{a_{x}}^{L} \frac{d x}{U_{1}^{2}(x,\langle\eta\rangle)}\left[u_{1}(x,\langle\eta\rangle)+g(x,\langle\eta\rangle) \eta^{\prime}\right]
\end{aligned}
$$

where

$$
g(x,\langle\eta\rangle)=\left.\frac{\partial\left[U_{1}(x, \eta)\right]}{\partial \eta}\right|_{\eta=\langle\eta\rangle}, \eta^{\prime},
$$

is the fluctuations of $\eta, U_{i}(x, \eta)$ and $u_{i}(x, \eta)(i=1,2)$ are the mean and perturbation of the Lagrangian velocity, respectively. If the flow field is stationary, then $g$ will be zero, and (23) and (24) return to the Dagan's results [Dagan, 1982; 1984]. From (24), we can obtain the covariance of $\tau(L ; \mathbf{a})$ as

$$
\begin{aligned}
& \sigma_{\tau_{1} \tau_{2}}(L, a ; L, b)=\int_{a_{x}}^{L} \int_{b_{x}}^{L} \frac{d x_{1} d x_{2}}{U_{1}^{2}\left(x_{1},\left\langle\eta_{1}\right\rangle\right) U_{2}^{2}\left(x_{2},\left\langle\eta_{2}\right\rangle\right)} \\
& \quad\left[\left\langle u_{1}\left(x_{1},\left\langle\eta_{1}\right\rangle\right) u_{2}\left(x_{2},\left\langle\eta_{2}\right\rangle\right)\right\rangle\right. \\
& \quad+g\left(x_{1},\left\langle\eta_{1}\right\rangle\right)\left\langle u_{1}\left(x_{2},\left\langle\eta_{2}\right\rangle\right) \eta_{1}^{\prime}\left(x_{1},\left\langle\eta_{1}\right\rangle\right)\right\rangle \\
& \quad+g\left(x_{2},\left\langle\eta_{2}\right\rangle\right)\left\langle u_{1}\left(x_{1},\left\langle\eta_{1}\right\rangle\right) \eta_{2}^{\prime}\left(x_{2},\left\langle\eta_{2}\right\rangle\right)\right\rangle \\
& \left.\quad+b_{1}\left(x_{1},\left\langle\eta_{1}\right\rangle\right) b_{1}\left(x_{2},\left\langle\eta_{2}\right\rangle\right)\left\langle\eta_{1}^{\prime}\left(x_{1},\left\langle\eta_{1}\right\rangle\right) \eta_{2}^{\prime}\left(x_{2},\left\langle\eta_{2}\right\rangle\right)\right\rangle\right]
\end{aligned}
$$

The statistical moments of $\eta$, to the first order, are obtained as

$$
\begin{aligned}
\frac{d\langle\eta(x ; \mathbf{a})\rangle}{d x}= & \frac{U_{2}(x,\langle\eta\rangle)}{U_{1}(x,\langle\eta\rangle)} \\
\frac{d \eta^{\prime}(x ; \mathbf{a})}{d x}= & A(x,\langle\eta\rangle) u_{1}(x,\langle\eta\rangle)+B(x,\langle\eta\rangle) u_{2}(x,\langle\eta\rangle) \\
& +C(x,\langle\eta\rangle) \eta^{\prime}(x ; \mathbf{a})
\end{aligned}
$$


where

$$
\begin{aligned}
& A(x,\langle\eta\rangle)=-\frac{U_{2}(x,\langle\eta\rangle)}{U_{1}^{2}(x,\langle\eta\rangle)} \\
& B(x,\langle\eta\rangle,\langle\xi\rangle)=\frac{1}{U_{1}(x,\langle\eta\rangle,\langle\xi\rangle)} \text { and } \\
& C(x,\langle\eta\rangle)=\left.\frac{\partial U_{2}(x, \eta)}{\partial \eta}\right|_{\eta=\langle\eta\rangle}-\left.\frac{U_{2}(x,\langle\eta\rangle)}{U_{1}(x,\langle\eta\rangle)} \frac{\partial U_{1}(x, \eta)}{\partial \eta}\right|_{\eta=\langle\eta\rangle}
\end{aligned}
$$

Multiplying (27) by $\eta^{\prime}\left(x_{2} ; \mathbf{b}\right)$, we obtain

$$
\begin{aligned}
\frac{d\left\langle\eta^{\prime}\left(x_{1} ; \mathbf{a}\right) \eta^{\prime}\left(x_{2} ; \mathbf{b}\right)\right\rangle}{d x_{1}}= & A\left(x_{1},\left\langle\eta_{1}\right\rangle\right)\left\langle u_{1}\left(x_{1},\left\langle\eta_{1}\right\rangle\right) \eta^{\prime}\left(x_{2} ; \mathbf{b}\right)\right\rangle \\
& +B\left(x_{1},\langle\eta\rangle\right)\left\langle u_{2}\left(x_{1}\langle\eta\rangle\right) \eta^{\prime}\left(x_{2} ; \mathbf{b}\right)\right\rangle \\
& +C\left(x_{1},\left\langle\eta_{1}\right\rangle\right)\left\langle\eta^{\prime}\left(x_{1} ; \mathbf{a}\right) \eta^{\prime}\left(x_{2} ; \mathbf{b}\right)\right\rangle
\end{aligned}
$$

with the initial condition (here we assume the initial positions of parcels are deterministic)

$$
\left.\left\langle\eta^{\prime}\left(x_{1} ; \mathbf{a}\right) \eta^{\prime}\left(x_{2} ; \mathbf{b}\right)\right\rangle\right|_{x_{1}=0 \text { or } x_{2}=0}=0
$$

Similarly, we can also obtain the expressions of $\left\langle u_{1} \eta^{\prime}\right\rangle$ and $\left\langle u_{2} \eta^{\prime}\right\rangle$. The velocity correlations, $\left\langle u_{i} u_{j}\right\rangle=$, can be obtained from the flow equations and are treated as known quantities or input data here. Therefore, there are three differential equations for the three unknowns, $\left\langle\eta^{\prime} \eta^{\prime}\right\rangle\left\langle u_{1} \eta^{\prime}\right\rangle$, and $\left\langle u_{2} \eta^{\prime}\right\rangle$, so the equations are solvable. Owing to the complexity of these equations, we solve them numerically.

From (24), the joint moments $\left\langle\tau^{\prime}\left(x_{1} ; \mathbf{a}\right) \eta^{\prime}\left(x_{2} ; \mathbf{b}\right)\right\rangle$ is obtained as

$$
\begin{aligned}
\left\langle\tau^{\prime}\left(x_{1} ; \mathbf{a}\right) \eta^{\prime}\left(x_{2} ; \mathbf{b}\right)\right\rangle= & -\int_{a_{x}}^{x_{1}} \frac{d_{\chi}}{U_{1}^{2}(\chi,\langle\eta\rangle)}\left[\left\langle u_{1}(\chi,\langle\eta\rangle) \eta^{\prime}\left(x_{2} ; \mathbf{b}\right)\right\rangle\right. \\
& \left.+g(\chi,\langle\eta\rangle,\langle\xi\rangle)\left\langle\eta^{\prime}(\chi ; \mathbf{a}) \eta^{\prime}\left(x_{2} ; \mathbf{b}\right)\right\rangle\right]
\end{aligned}
$$

From (23) and (26), a parcel's mean movements in longitudinal and transverse direction can be calculated through a finite difference numerical method. The 
method for computing the first moments is similar to the numerical particle tracking method [Hassan et al., 1998]. From (25) and (29), $\left\langle\tau^{\prime} \tau^{\prime}\right\rangle$ and $\left\langle\tau^{\prime} \eta^{\prime}\right\rangle$ can be obtained based on the calculated results of $\left\langle\eta^{\prime} \eta^{\prime}\right\rangle,\left\langle u_{1} \eta^{\prime}\right\rangle$ and $\left\langle u_{2} \eta^{\prime}\right\rangle$.

We assume that for each parcel, its $\tau$ and $\eta$ obey lognormal and normal distributions, respectively, their joint PDFs $f_{1}[\tau(x, a), \eta(x, \mathbf{a})]$ for one particle and $f_{2}\left[\tau_{1}(x, a), \eta_{1}(x, \mathbf{a}) ; \tau_{2}(x, b), \eta_{2}(x, \mathbf{b})\right]$ for two particles can be directly related to the covariances of $\tau^{\prime}$ and $\eta^{\prime}$. From (20)-(22), one may see that the solute flux is the summation or integral of all parcels' distributions. Therefore, even if one parcel is assumed to satisfy lognormal and normal distributions in the longitudinal and transverse directions, respectively, the distribution of all plume (the summation of all parcels) in longitudinal and transverse directions could be quite abnormal in some cases due to the variations of the mean movements of the parcels and variances about the means. In the next sections, the methods introduced in this section will be applied to study groundwater flow and solute transport in multi-scale media.

\section{Illustrative example: flow and solute transport in a bi-model medium}

In this section we use a case study to investigate the influences of the two scale stochastic processes of a multi-scale medium on the prediction of groundwater flow and solute transport. The study domain is a square with the size $10 \times 10 \mathrm{~m}^{2}$, shown in figure 1. The medium is composed of a lot of small pieces of the two materials, and the two materials are uniformly distributed with each other, like many puzzles, which is so-called bimodal medium. The distributions of two materials are stationary. This phenomenon can be observed in many geological media, such as the lenses of sand, silt and clay inter-bedded with each other in the alluvial sediments, or the various lava mixed together in some igneous rocks. We denote $P(\mathbf{x})=p 1(\mathbf{x})$ to represent the possibility of finding material 1 in the point $x$. Since the two materials are uniformly distributed, $P(x)$ also represents the percentage of the material 1 in the whole domain. We use $\lambda_{I}$ to represent the correlation length of the geometry indicator $I(x)$.

We impose constant heads on the vertical sides of the domain, $H=10.0 \mathrm{~m}$ on the left side and $H=0.0 \mathrm{~m}$ on the right side, and assume the other two sides are impermeable boundaries. A finite difference method is implemented for the 


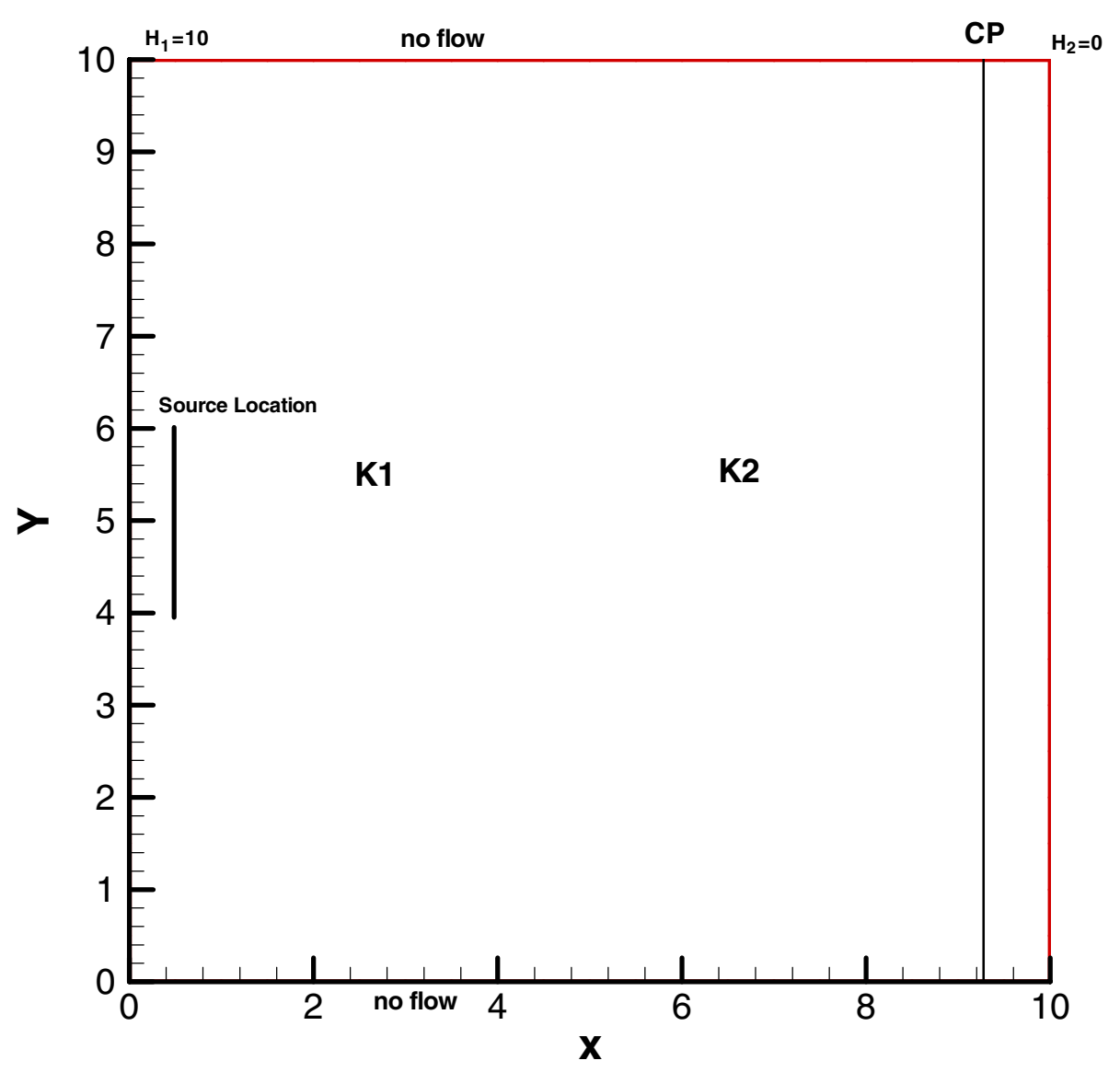

Figure 1 - Sketch of the study medium.

various calculations for flow and transport. The whole domain is uniformly discretized into $40 \times 40$ square elements with a cell size of $0.25 \times 0.25 \mathrm{~m}^{2}$. One unit of solute mass is uniformly distributed in the source line.

Figure 2 shows the covariance of log-hydraulic conductivity with the reference point at $(5.0,5.0)$ under $P(x)=0.0,0.5$ and 1.0. In this case, we choose $\sigma_{Y_{1}}^{2}=0.4, \sigma_{Y_{2}}^{2}=0.6, \lambda_{1}=0.5 \mathrm{~m}, \lambda_{2}=1.0 \mathrm{~m}$ and $\lambda_{I}=4.0 \mathrm{~m}$. It is shown from the figure that in comparison with a single material, the mixture of the two materials significantly increases the covariance of the log-hydraulic conductivity in the whole domain, and the conductivity is correlated in a much longer range.

Figures $3 \mathrm{a}$ and $3 \mathrm{~b}$ present the distribution of the mean hydraulic head and the variance of the hydraulic head, respectively, along the longitudinal cross-line $x_{2}=5.0 m$ with $P(x)=0.0,0.3,0.5,0.7$ and 1.0. It is shown from figure 3a that the mean head will not be influenced by the variation of $P(x)$, but the head variance significantly increase with the mixture of the two materials in 


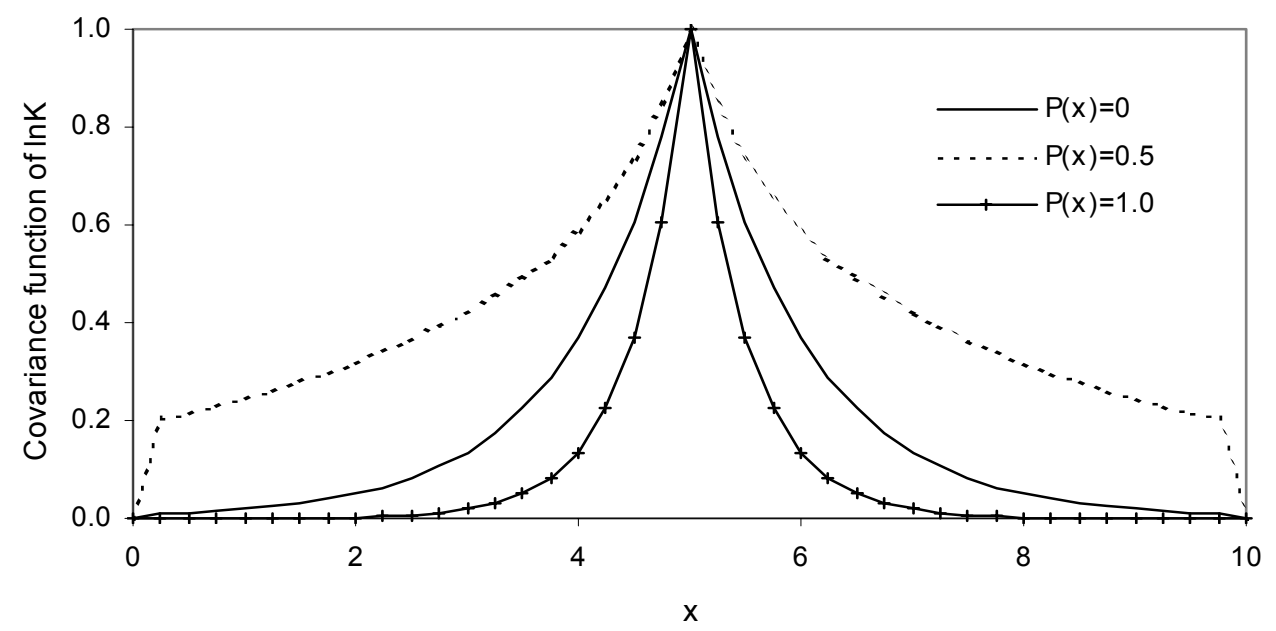

Figure 2 - Covariance function of log hydraulic conductivity for the longitudinal cross-section with the reference point $(5.0,5.0)$ for various degrees of probability $P(x)=0,0.5$ and 1 .

comparison with those of one materials. When $P(\mathbf{x})=0.5$, which means the compositions of two materials are equal, the predicted variance of hydraulic head reaches its maximum in this case.

Figures 4a,b show the breakthrough curves of expected values and variances of $Q$ with $P(x)=0.0,0.3,0.5,0.7$ and 1.0, respectively. The breakthrough curves of $\langle Q\rangle$ change with the variation of $P(x)$, but bounded in the two onescale cases, $P(x)=0.0$ and 1.0. In comparison with the results of one-scale cases, the breakthrough curves in the two-scale cases have longer tails and more dispersion. The $\sigma_{Q}$ curves have the similar shapes to $\langle Q\rangle$ 's, but $\sigma_{Q}$ reaches its maximum when $P(x)=0.5$. Figures 5a,b shows the influences of $P(x)$ on $q$ at the center point of the control line. The influences are similar to those on $\langle Q\rangle$ and $\sigma_{Q}$.

The above studies in this subsection focus on the influences of $P(x)$ on flow and transport, and other parameters are fixed. In the two-scale heterogeneous medium, there are other heterogeneities that will also affect the flow and transport processes. In the small scale, the heterogeneity results from the spatial variation of conductivity field within each material. This heterogeneity is statistically characterized by the variance, correlation function and correlation length of hydraulic conductivity of every material in the medium. In the large heterogeneous scale, the heterogeneity is from the different mean values of hydraulic conductivity of 

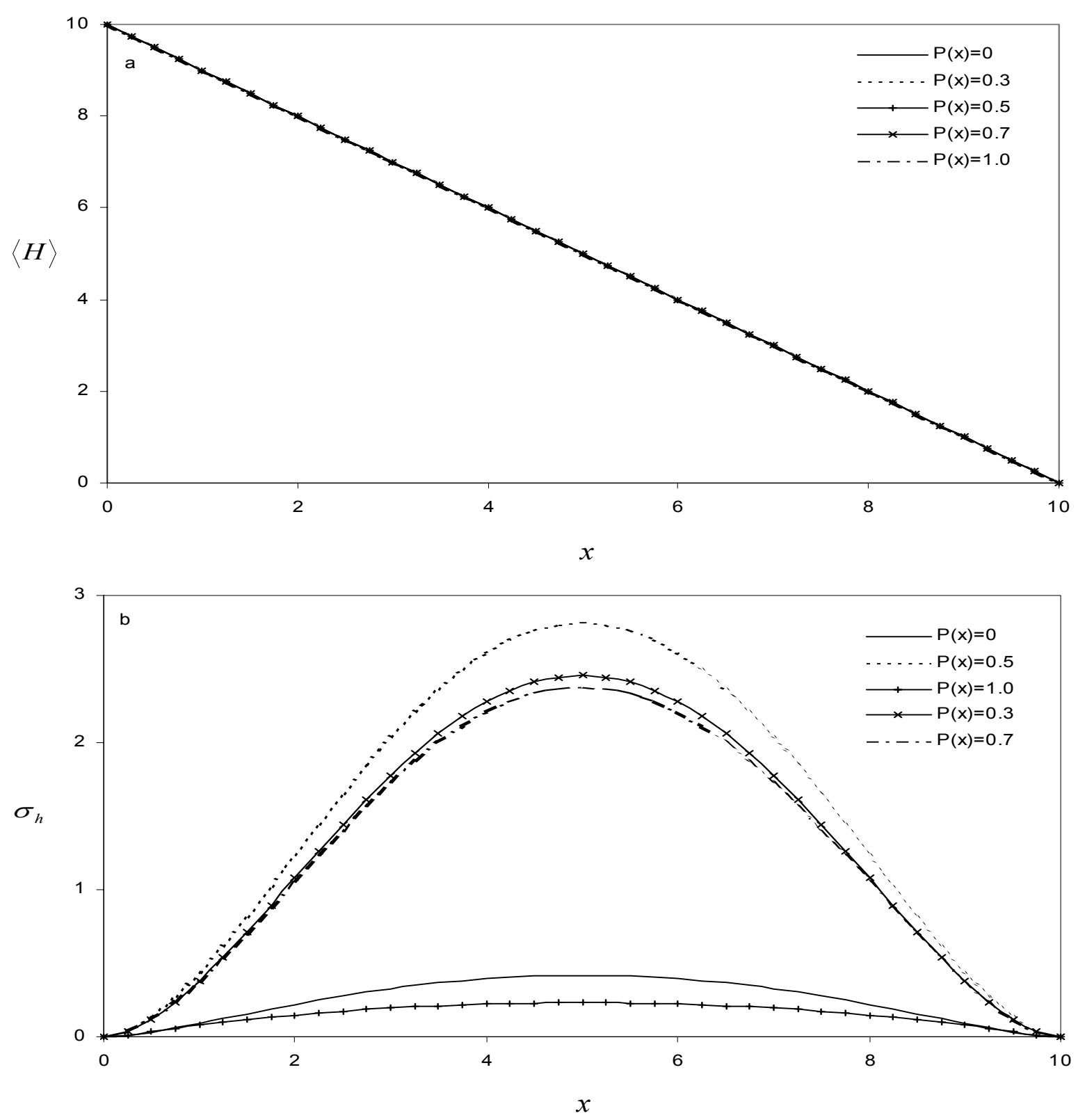

Figure 3 - Distribution of (a) the mean hydraulic head, and (b) the standard deviation of the hydraulic head along the longitudinal cross-section $x_{2}=5.0 \mathrm{~m}$ for various degrees of probability $P(\mathbf{x})=0,0.3,0.5,0.7$ and 1.0 .

various materials, the compositions of various materials (which is described by $\left.P_{i}(x)\right)$ and the distribution of the materials, which is described by the correlation function and correlation length of the indicate $I(x)$. Here, we use 8 cases, cases 1-8, with various combinations of parameter values, shown in table 1 , to investigate the influences of the various uncertainty factors on flow and transport. Since the effects of $P(x)$ on flow and transport have already been studied above, 

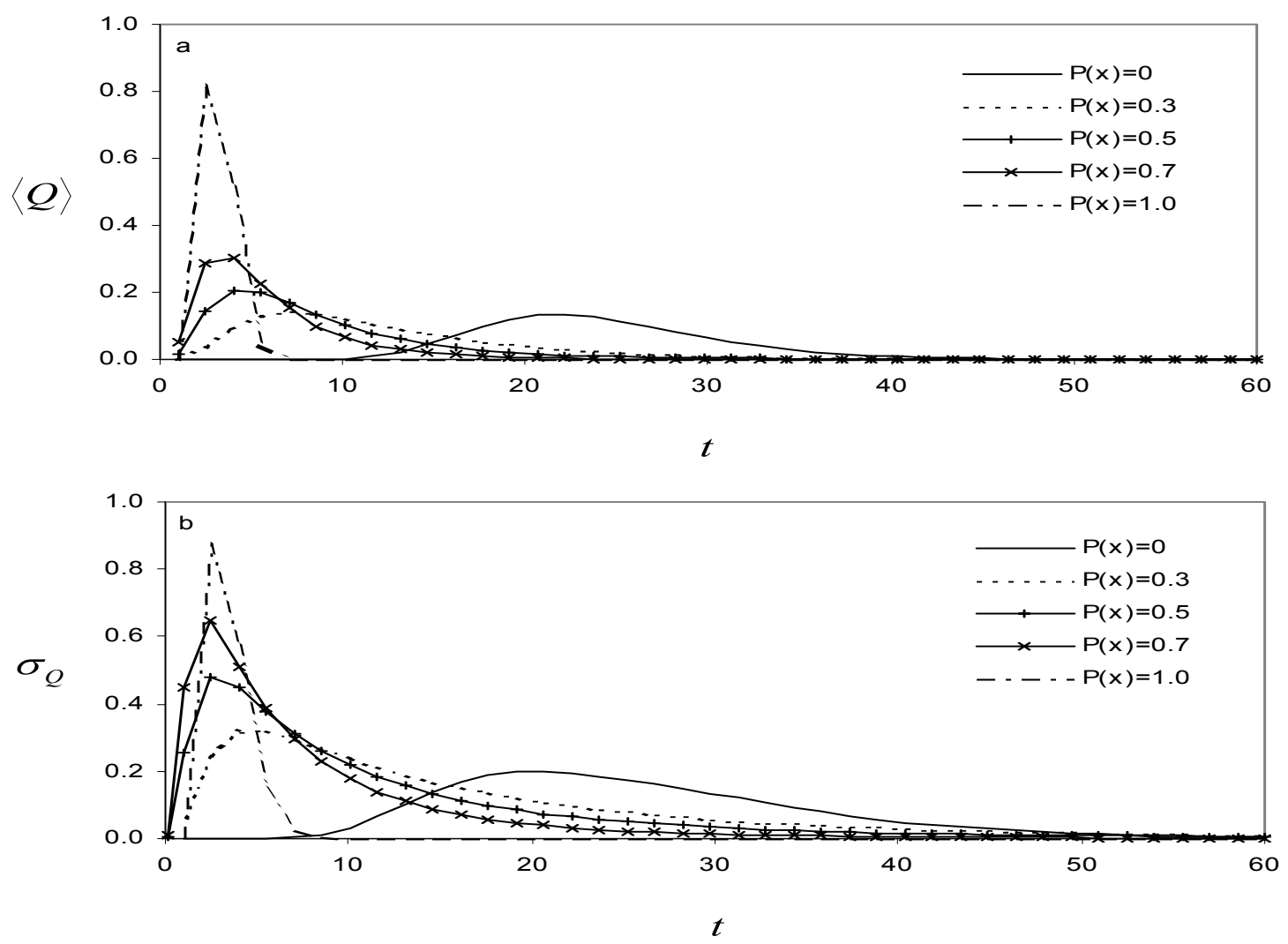

Figure 4 - Breakthrough curves of the total solute flux through the control plane for various degrees of probability $P(x)=0,0.3,0.5,0.7$ and 1.0 , (a) the expected value $\langle Q\rangle$, (b) the standard deviation $\sigma_{Q}$.

here we fix its value to be 0.5 . In these case studies, we also assume that the $\log$ conductivity field in each material and the indicator function $I(x)$ all have exponential auto-covariances. The parameters in the last two columns, i.e., $\langle Y\rangle$ and $\sigma_{Y}^{2}$, are the effective means and variances of the log hydraulic conductivity of the composite medium, which are calculated from Eqs. (2) and (9).

The selection of the eight cases is under the consideration: cases 1, 3 and 5 are used to study the effect of the difference between $\left\langle Y_{1}\right\rangle$ and $\left\langle Y_{2}\right\rangle$ on flow and transport processes; cases 3, 7 and 8 are grouped to investigate the effects of the various correlation lengths on flow and transport prediction; the three pairs of case 1 and 2, case 3 and 4, case 5 and case 6 are designed to differentiate the effects of variances on the two scales on prediction of flow and transport. In all these case studies, (external) hydraulic boundary conditions and the locations of the solute source and $\mathrm{CP}$ are all same as those shown in figure 1.

Cases 1-6 are grouped to study the influences of the larger-scale heterogeneity, 

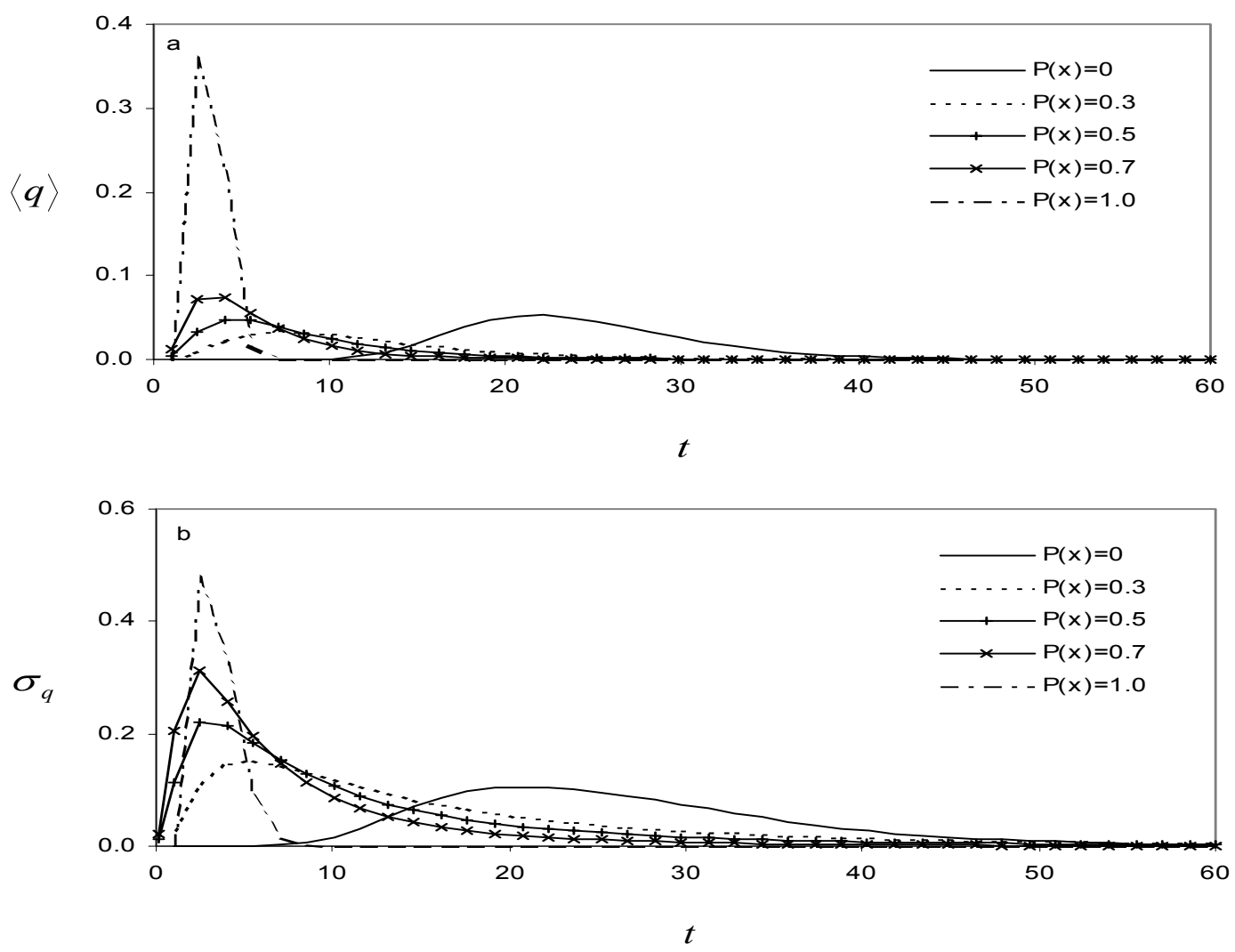

Figure 5 - Breakthrough curves of the solute flux through the control plane for various degrees of probability $P(x)=0.0,0.3,0.5,0.7$ and 1.0 , (a) the expected value $\langle q\rangle$, (b) the standard deviation $\sigma_{q}$.

\begin{tabular}{l|c|c|c|c|c|c|c|c|c}
\hline Parameter & $\left\langle Y_{1}\right\rangle$ & $\left\langle Y_{2}\right\rangle$ & $\sigma_{Y_{1}}^{2}$ & $\sigma_{Y_{2}}^{2}$ & $\lambda_{1}$ & $\lambda_{2}$ & $\lambda_{I}$ & $\langle Y\rangle$ & $\sigma_{Y}^{2}$ \\
\hline Case 1 & 1.0 & -1.0 & 1.0 & 1.0 & 0.5 & 1.0 & 4.0 & 0.0 & 2.0 \\
Case 2 & 1.0 & -1.0 & 0.0 & 0.0 & 0.5 & 1.0 & 4.0 & 0.0 & 1.0 \\
Case 3 & 2.0 & -2.0 & 1.0 & 1.0 & 0.5 & 1.0 & 4.0 & 0.0 & 5.0 \\
Case 4 & 2.0 & -2.0 & 0.0 & 0.0 & 0.5 & 1.0 & 4.0 & 0.0 & 4.0 \\
Case 5 & 3.0 & -3.0 & 1.0 & 1.0 & 0.5 & 1.0 & 4.0 & 0.0 & 10.0 \\
Case 6 & 3.0 & -3.0 & 0.0 & 0.0 & 0.5 & 1.0 & 4.0 & 0.0 & 9.0 \\
Case 7 & 2.0 & -2.0 & 1.0 & 1.0 & 2.0 & 2.0 & 4.0 & 0.0 & 5.0 \\
Case 8 & 2.0 & -2.0 & 1.0 & 1.0 & 2.0 & 2.0 & 2.0 & 0.0 & 5.0 \\
Case 9 & 0.5 & -0.5 & 0.4 & 0.2 & 0.5 & 0.5 & 1.5 & 0.0 & 0.55 \\
Case 10 & 1.0 & -1.0 & 0.4 & 0.2 & 0.5 & 0.5 & 1.5 & 0.0 & 1.3 \\
\hline
\end{tabular}

Table 1 - Parameter specifications for all cases $P(x)=0.5$. 


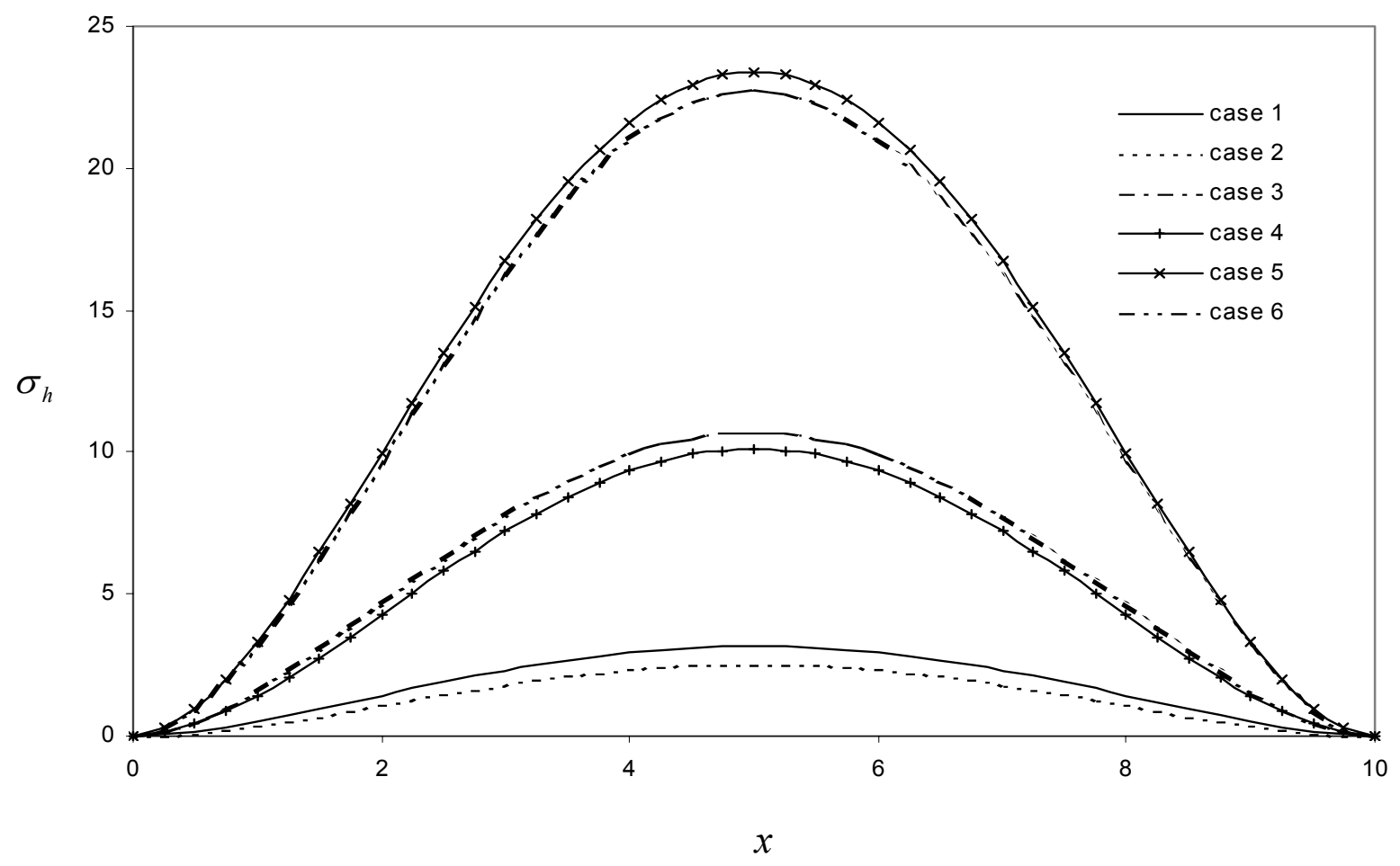

Figure 6 - Distribution of (a) the mean hydraulic head, and (b) the standard deviation of the hydraulic head along the longitudinal cross-section $x_{2}=5.0 \mathrm{~m}$ at $P(x)=0.5$ for cases $1,2,3,4,5$ and 6 , respectively.

$\left\langle Y_{1}\right\rangle-\left\langle Y_{2}\right\rangle$, and small-scale heterogeneity, $\sigma_{Y_{1}}^{2}$ and $\sigma_{Y_{2}}^{2}$, on hydraulic head variance and solute flux. Figures 6 shows the distribution of $\sigma_{h}^{2}$ along the longitudinal cross-line $x_{2}=5.0 \mathrm{~m}$ for the six cases. Figures $7 \mathrm{a}, \mathrm{b}$ shows the breakthrough curves of expected values and variances of total solute flux $Q$ through $\mathrm{CP}$ in the six cases. Figures 8a,b present the breakthrough curves of $\langle q\rangle$ and $\sigma_{1}^{2}$, respectively. It is shown from figures 6-8 that the variations of $\sigma_{Y_{1}}^{2}$ and $\sigma_{Y_{2}}^{2}$ hardly affect $\sigma_{h}^{2}$ and $\langle Q\rangle$, but influence $\sigma_{Q},\langle q\rangle$ and $\sigma_{q}^{2}$. However the influences are secondary to those caused by $\left\langle Y_{1}\right\rangle-\left\langle Y_{2}\right\rangle$. In these cases, the total variance, $\sigma_{Y}^{2}$, significantly influence the distribution of head variance, mean and variance of solute flux, and $\sigma_{Y}^{2}$ is largely contributed from the large-scale heterogeneity.

In the above study, the effects of $\left\langle Y_{1}\right\rangle-\left\langle Y_{2}\right\rangle, \sigma_{Y_{1}}^{2}, \sigma_{Y_{2}}^{2}$ and $\sigma_{Y}^{2}$ on flow and transport have been studies. Here, we would like to group cases 3, 7 and 8 to analyze the effects $?_{1}, ?_{2}$ and $?_{I}$ on the flow and transport. Figures 9 shows the distribution of $\sigma_{h}^{2}$ along the longitudinal cross-line $x_{2}=5.0 \mathrm{~m}$ for the three cases. In comparison of cases 3 and 7 , it is shown that $\sigma_{h}^{2}$ increases with the increases of $?_{1}$ and $?_{2}$. 

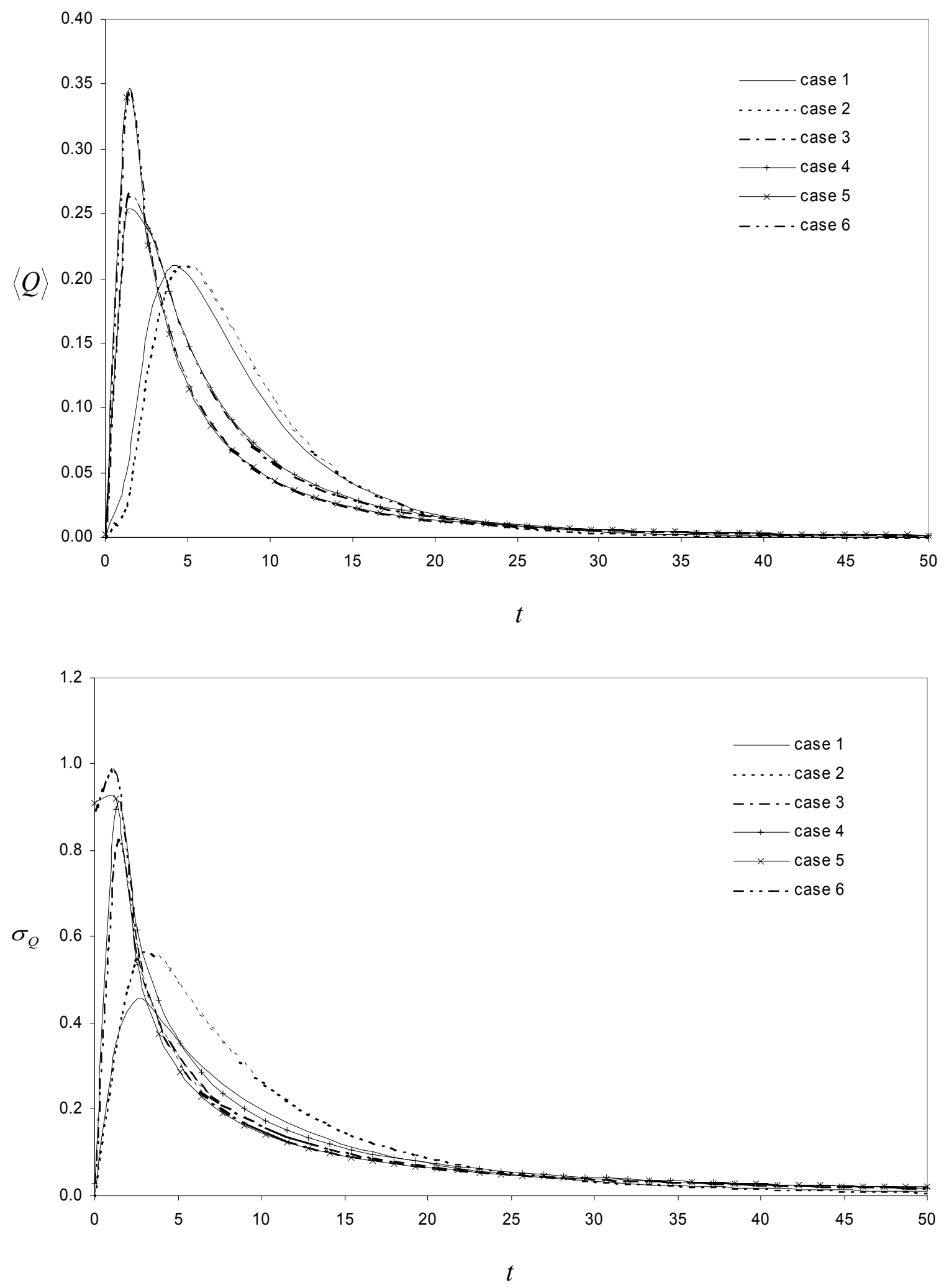

Figure 7 - Breakthrough curves of the total solute flux through the control plane at $P(x)=0.5$ for cases $1,2,3,4,5$ and 6 , respectively, (a) the expected value $\langle Q\rangle$, (b) the standard deviation $\sigma_{Q}$. 

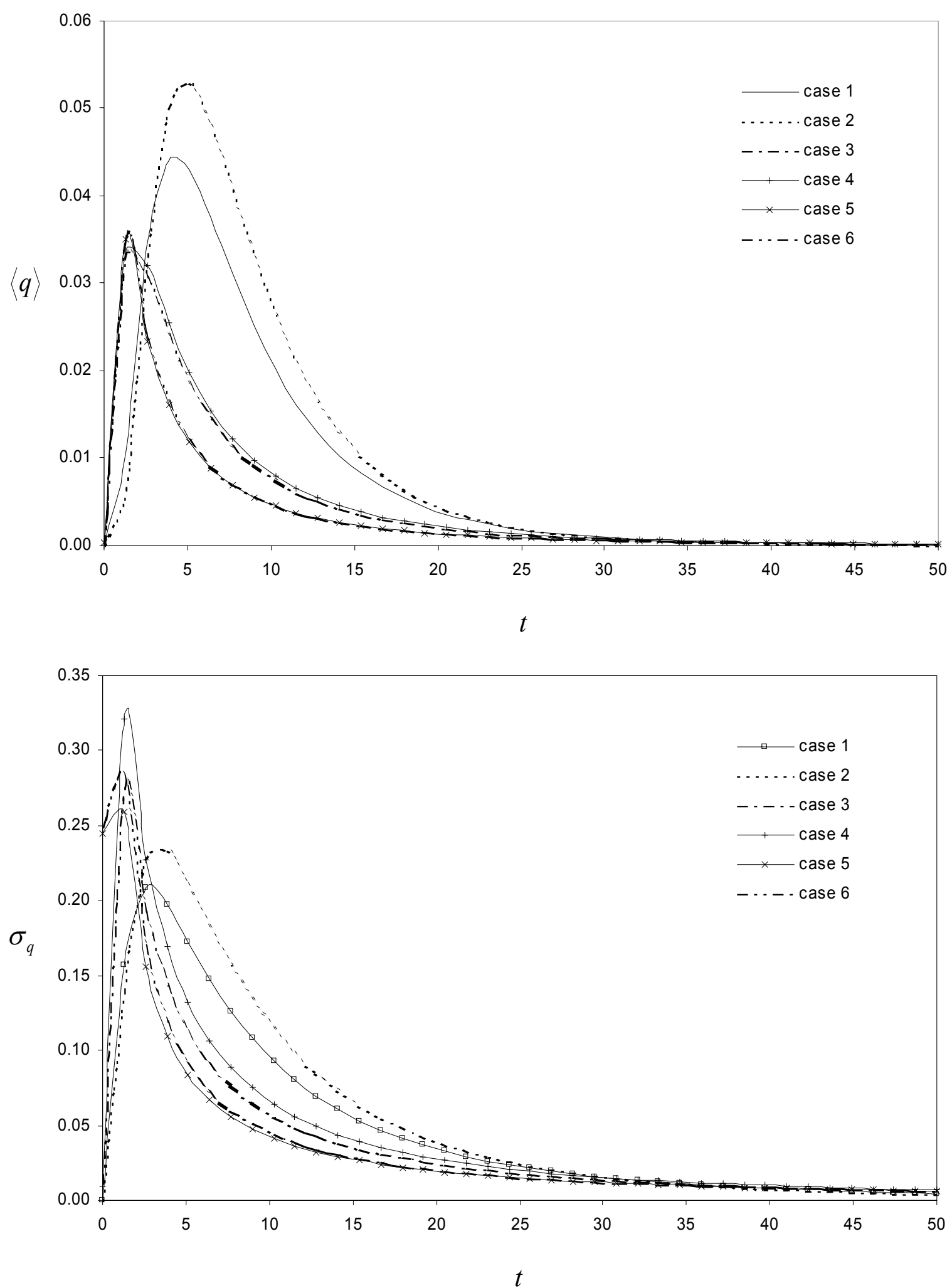

Figure 8 - Breakthrough curves of the total solute flux through the control plane at $P(x)=0.5$ for cases $1,2,3,4,5$ and 6 , respectively, (a) the expected value $\langle q\rangle$, (b) the standard deviation $\sigma_{q}$. 


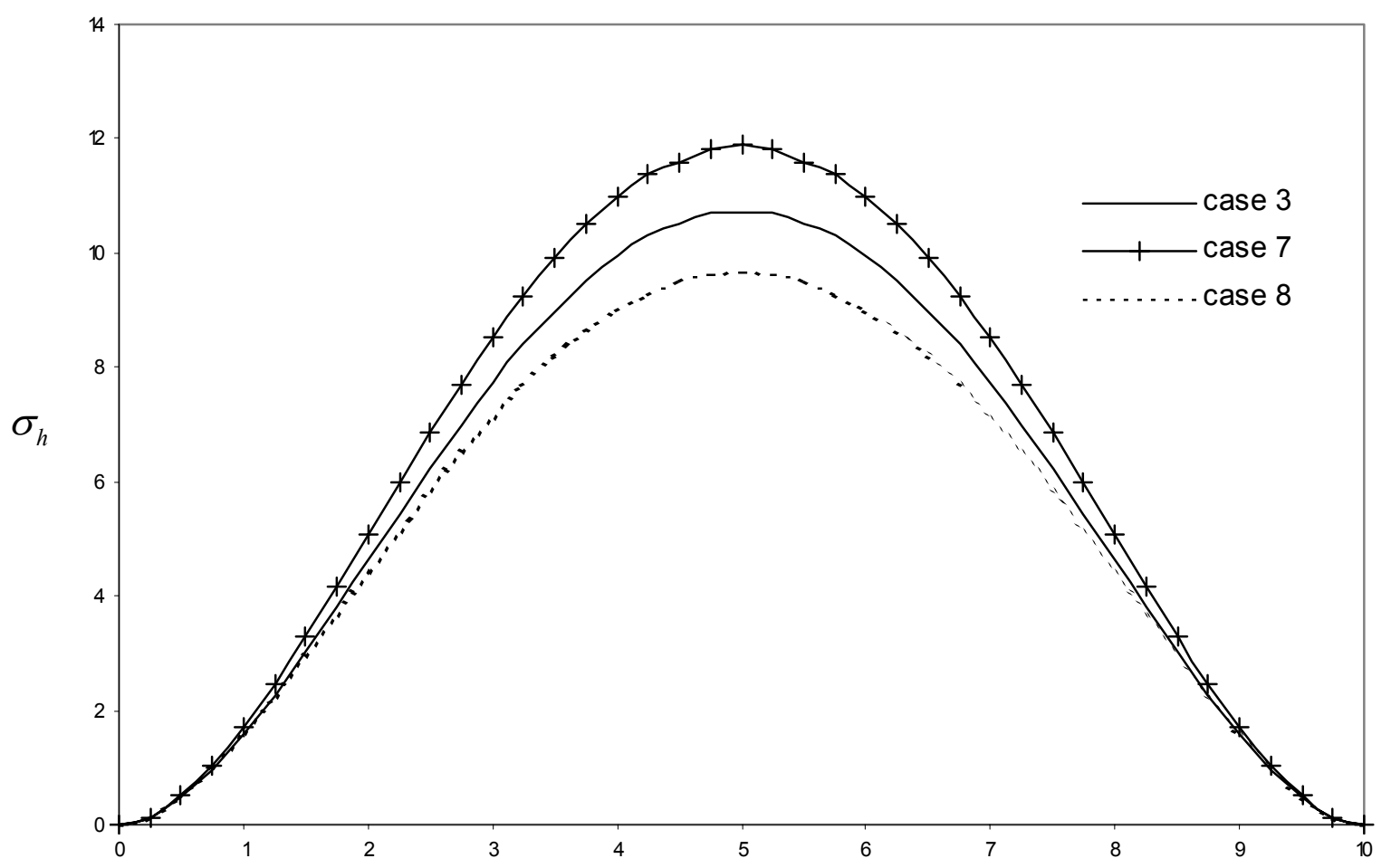

Figure 9 - Distribution of the standard deviation of the hydraulic head along the longitudinal cross-section $x_{2}=5.0 \mathrm{~m}$ at $P(x)=0.5$ for cases 3,7 and 8 , respectively.

Similarly, from the results of case 7 and 8 , one case see that $\sigma_{h}^{2}$ increase with the increase of $\lambda_{I}$.

Figures 10a,b shows the breakthrough curves of expected values and variances of total solute flux $Q$ through CP for the three cases. It is shown from the figures that with the increases of $?_{1}, ?_{2}$ and $\lambda_{I},\langle Q\rangle$ curve has an earlier breakthrough and the high peak, but the tail does not change. The increases of $?_{1}, ?_{2}$ and $?_{I}$ also lead to the increase of $\sigma_{Q}^{2}$. Figures $11 \mathrm{a}, \mathrm{b}$ present the breakthrough curves of $\langle q\rangle$ and $\sigma_{q}^{2}$, respectively. $\langle q\rangle$ and $\sigma_{q}^{2}$ curves are quite different from their counterparts of $\langle Q\rangle$ and $\sigma_{Q}^{2}$, which is caused by the transverse dispersion.

\section{Comparison of NMM and Monte Carlo simulation results}

In the above study, the NMM has been developed to study the groundwater flow and solute transport in multi-scale, heterogeneous porous media. The newly developed method needs to compare with a well-developed method to verify 

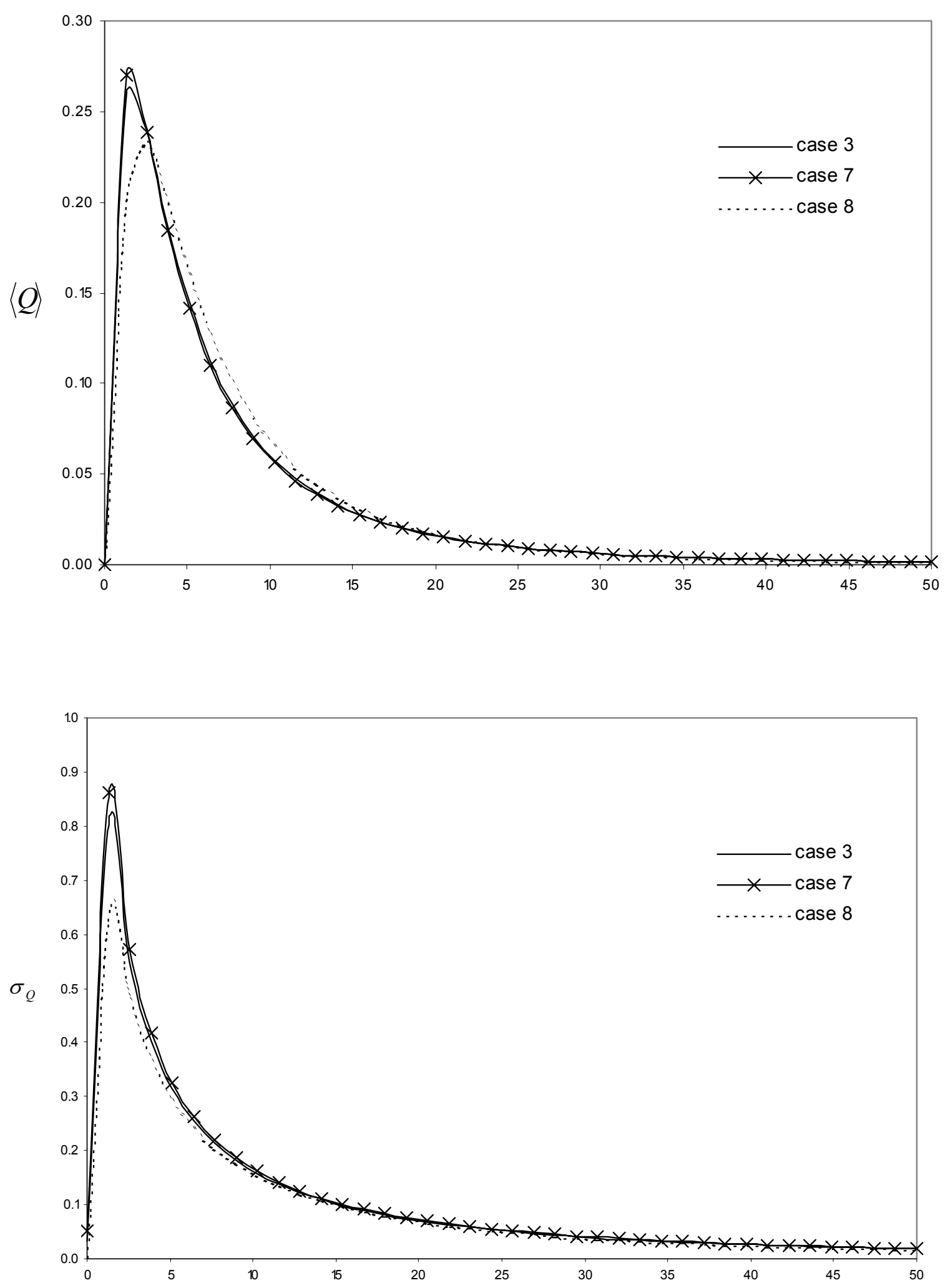

Figure 10 - Breakthrough curves of the total solute flux through the control plane at $P(x)=0.5$ for cases 3,7 and 8 , respectively, (a) the expected value $\langle Q\rangle$, (b) the standard deviation $\sigma_{Q}$. 

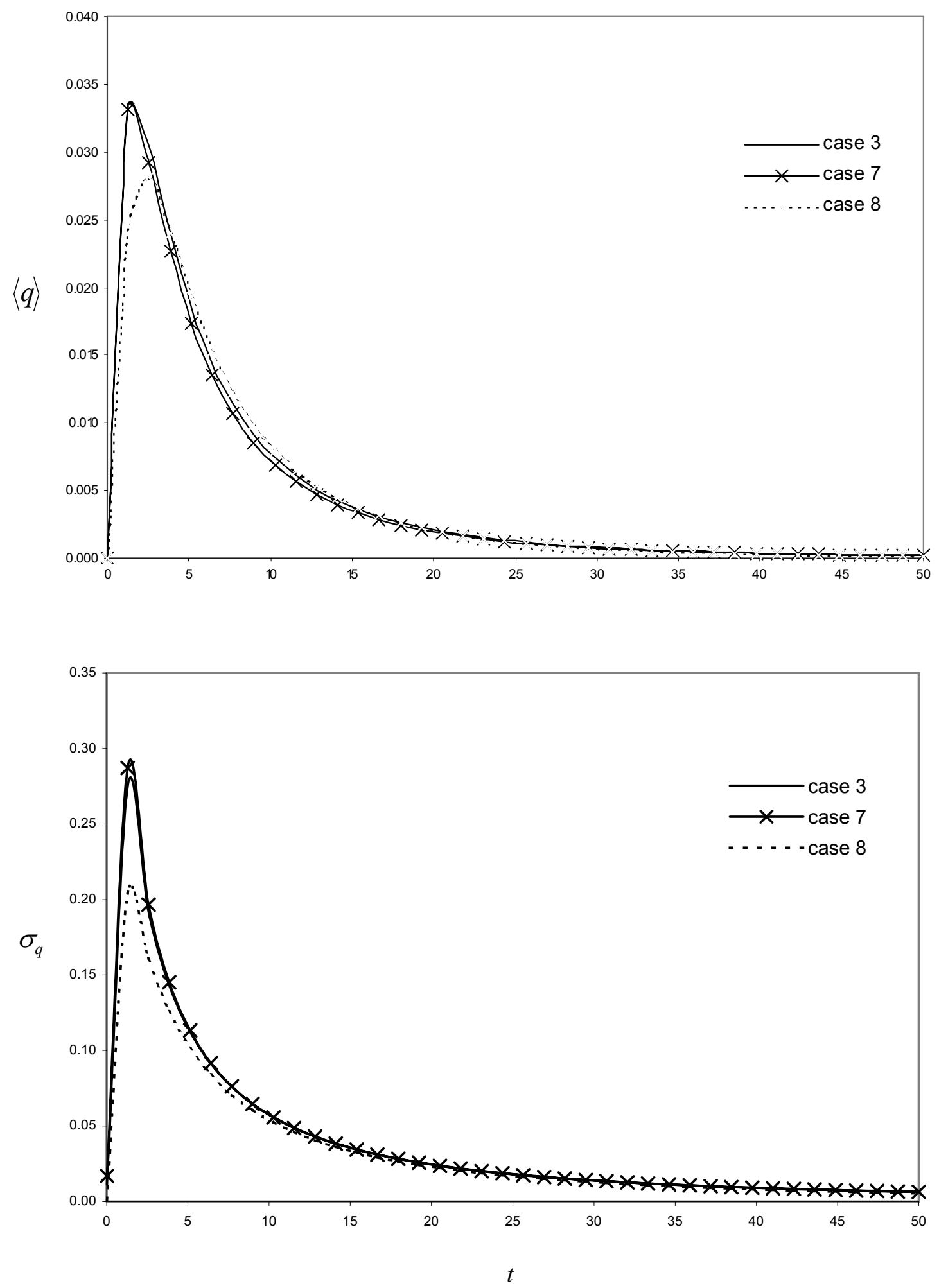

Figure 11 - Breakthrough curves of the total solute flux through the control plane at $P(x)=0.5$ for cases 3,7 and 8 , respectively, (a) the expected value $\langle q\rangle$, (b) the standard deviation $\sigma_{q}$. 
the calculation results. In this section, a Monte Carlo numerical simulation method is applied to check the results obtained by the NMM. This kind of study has been conducted for flow in multi-scale media [Winter et al., 2002; Lu and Zhang, 2002]. In this study, we only compare the transport results in a bimodal medium.

\subsection{Generation of multi-realizations of hydraulic conductivity field}

The study domain is the same as the one shown in figure 1 . The study domain is discretized into $100 \times 100$ square elements with a size of $0.1 \times 0.1 \lambda^{2}$. Two cases, cases 9 and 10 in table 1 , are chosen in this section. The assumptions for the bi-model conductivity field made here are the same as those made in section 2. A three-step method is used to generate the bi-model conductivity field. First, according to the conductivity parameters provided in table 1 for $Y_{1}$ and $Y_{2}, 3,000$ realizations of the conductivity field are generated for each of the two media with a Fast Fourier Transform method. Secondly, a Gaussian random field generator sisim from GSLIB [Deutsch, C. V., A. G. Journel, 1998] is applied to generate 3,000 realizations of the geometry indicator field with $\lambda_{I}=1.5 \lambda$ and $p_{1}=p_{2}=0.5$. Third, each indicator field is combined with one realization of $Y_{1}$ and $Y_{2}$ to form one realization of the bi-modal conductivity field. As an example, one conductivity realization for case 9 is shown in figure 12 .

Figures 13a,b show the histograms of the log hydraulic conductivity realizations for cases 9 and 10. It is shown from the two figures that the bi-model distribution appears only when the difference between $\left\langle Y_{1}\right\rangle$ and $\left\langle Y_{2}\right\rangle$ is large enough in comparison with $\sigma_{Y_{1}}^{2}$ and/or $\sigma_{Y_{2}}^{2}$.

\subsection{Groundwater flow and solute transport simulations}

The hydraulic boundary conditions are the same as those shown in figure 1, except $H_{1}=1.0$. The locations of source line and CP are the same as those shown in figure 1. For each realization of hydraulic conductivity field, a two-dimensional flow simulator based on the finite-difference method is used to obtain hydraulic head and groundwater velocity distributions in the study domain subjected to the boundary conditions. Then, a streamline, particle-tracking approach is applied 


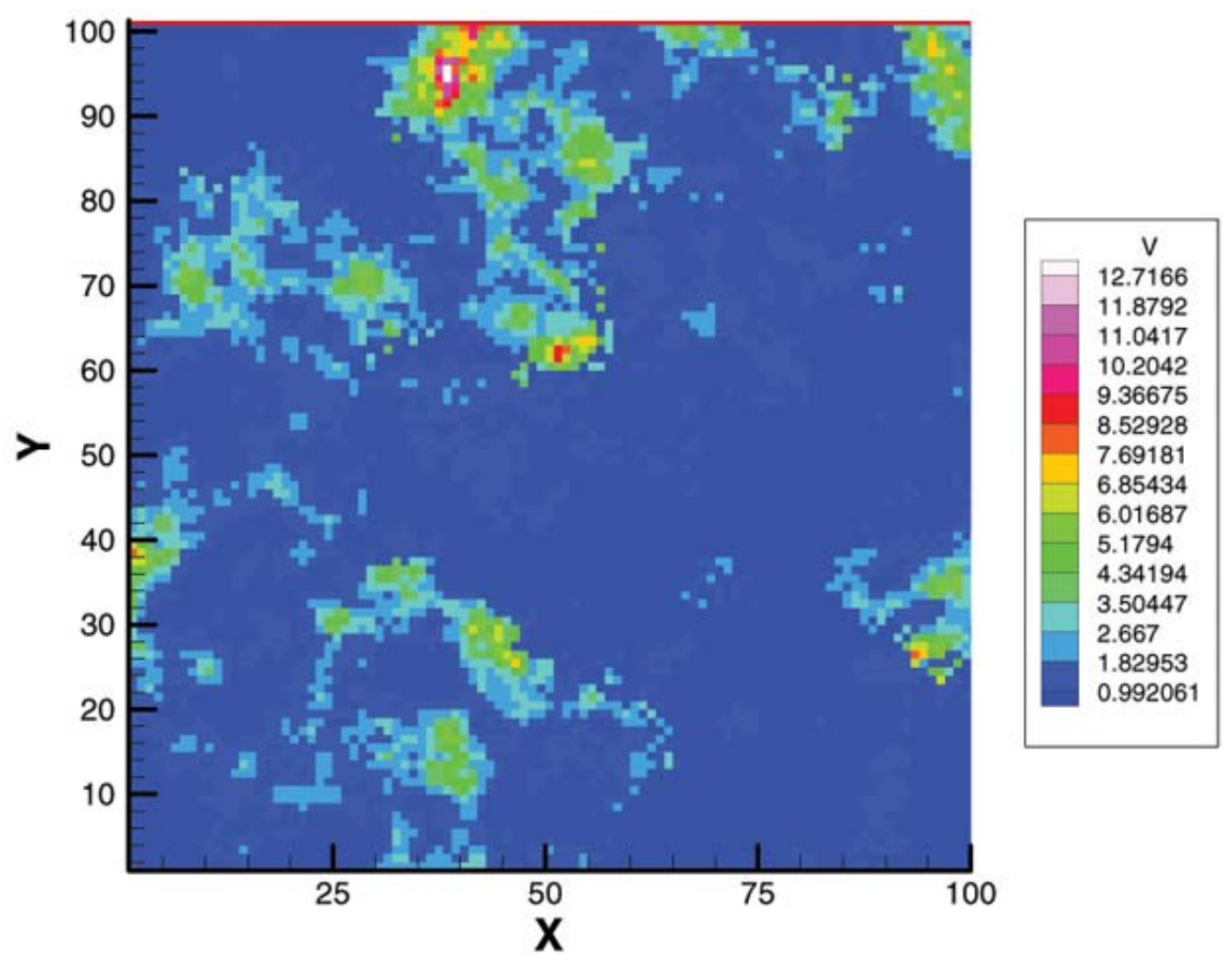

Figure 12 - One realization of bimodal conductivity field.

to simulate the movement of solute by a large number of particles. Therefore, the calculation of solute flux across the $\mathrm{CP}$ becomes the calculation of arrival times of individual particles reaching the $\mathrm{CP}$. The obtained multi-realizations of the solute flux curve are averaged over realizations to obtain the mean and variance of the solute flux.

Figures 14 and 15 show the results of the Monte Carlo simulation and NMM for two cases. Since the local dispersivity is not considered in the Monte Carlo simulation, the breakthrough curve is not smooth. For case 9, the calculation results of the two methods are almost identical. With the increase of the difference between $\left\langle Y_{1}\right\rangle$ and $\left\langle Y_{2}\right\rangle$, the NMM results will deviate from those of the Monte Carlo simulation, which are shown in Figure 10. In comparison with Monte Carlo results, the NMM method overestimate the peak value of $\langle Q\rangle$, but underestimate the extension of the tail. For the calculation of $\sigma_{Q}^{2}$, NMM curve is shifted right around peak value area in comparison with Monte Carlo simulation. This difference between NMM and Monte Carlo results may be due to the first-order accuracy of NMM, or/and insufficient realizations of Monte Carlo simulations. 


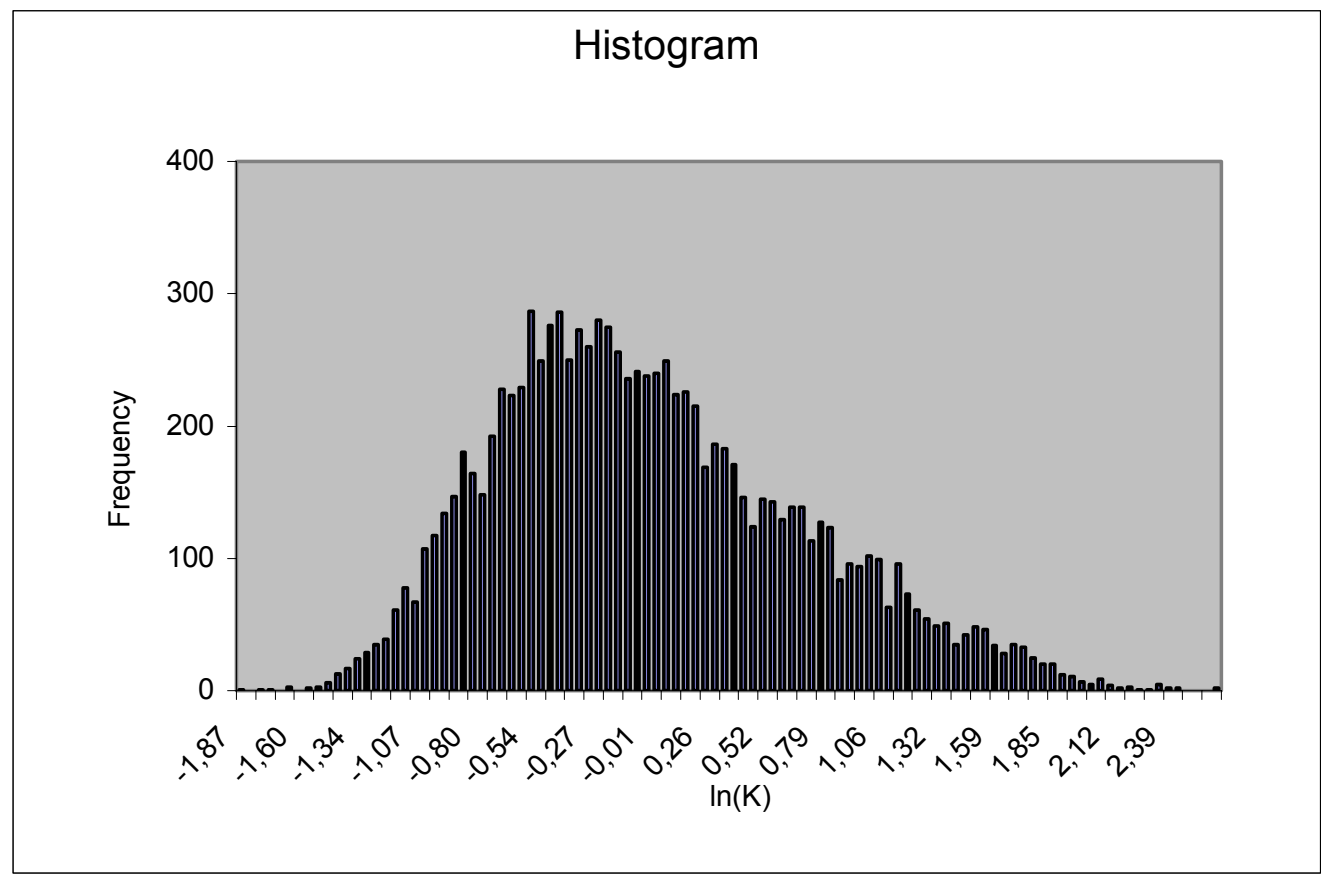

(a)

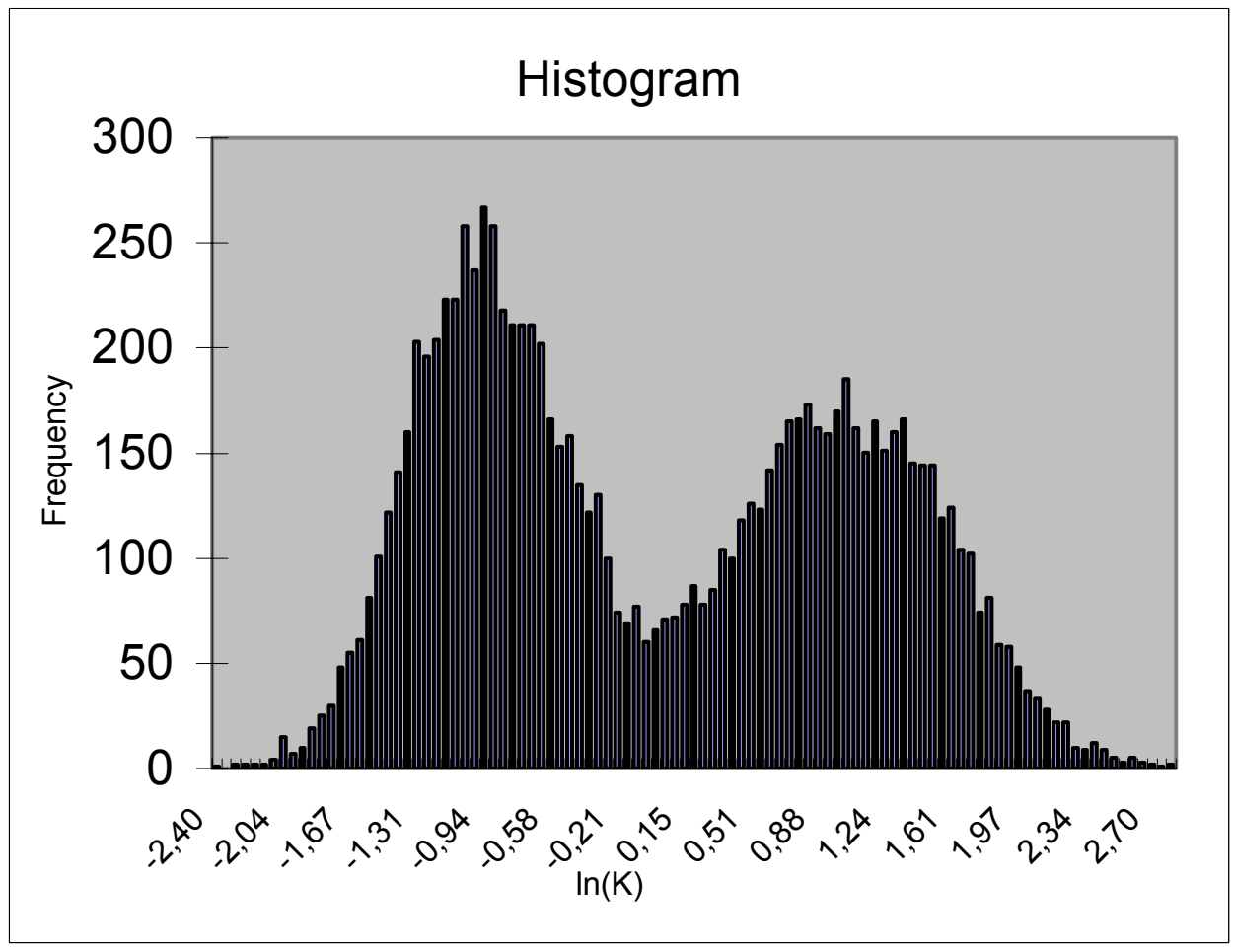

(b)

Figure 13 - Histogram of the log hydraulic conductivity, (a) case 9, (b) case 10. 
This issue needs to be addressed in future study. Generally speaking, NMM captures the dominate characteristics of the breakthrough curves as shown in Figure 14 for case 9, and Figure 15 for case 10.
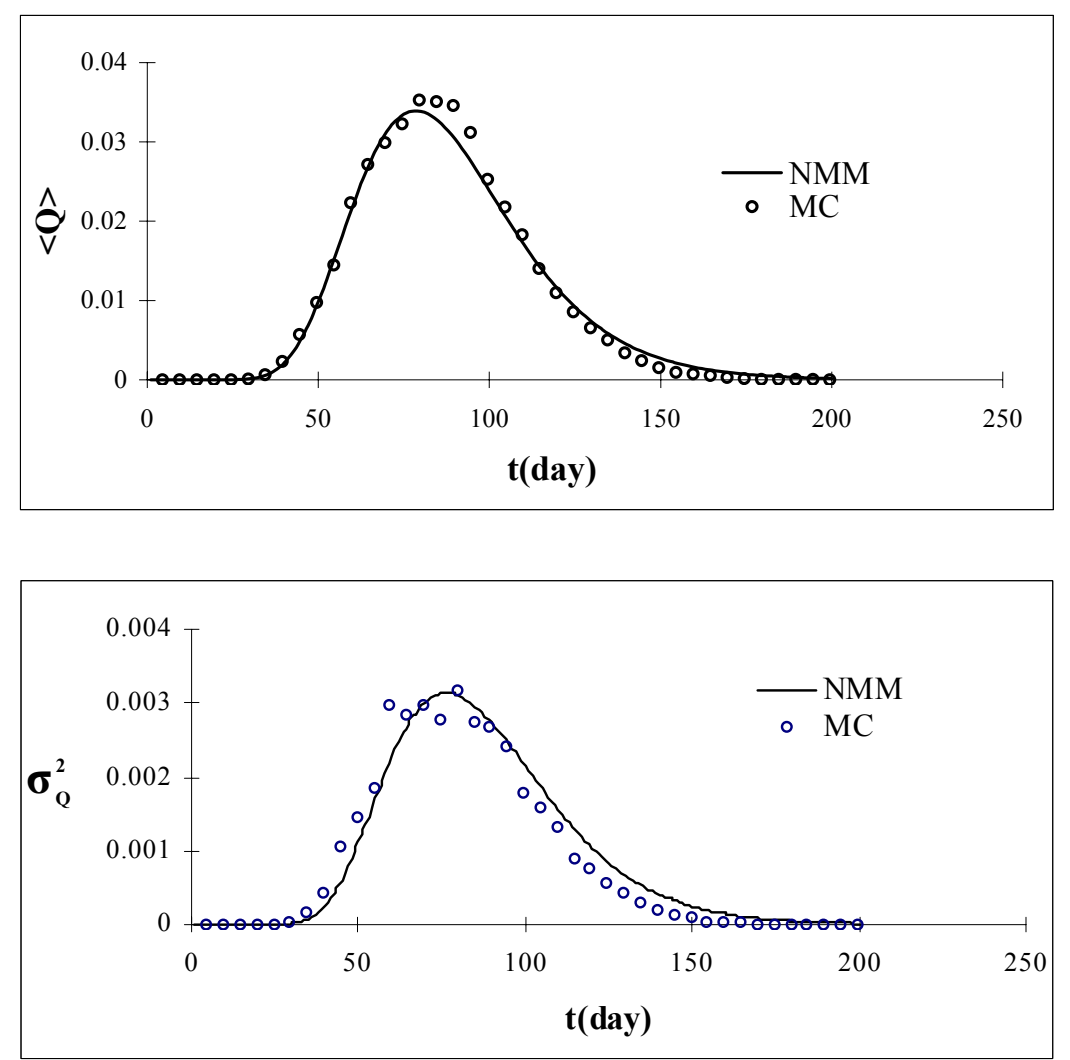

Figure 14 - Comparison of calculation results between Monte Carlo simulation and NMM method for case 9 .

\section{Summary and conclusions}

In this article, we study groundwater flow and solute transport in a multi-scale heterogeneous medium. The medium is composed of various materials, each of which may have its own mean, variance and correlation length of hydraulic conductivity. The distribution of materials in the medium is characterized by an indicator function. In this study, we first derived explicit expressions for the covariance function of the composite field in terms of the covariance of the indicator variables and the properties of the different composite materials. The expression is so general that many previous study results become the specific cases of the expression. 

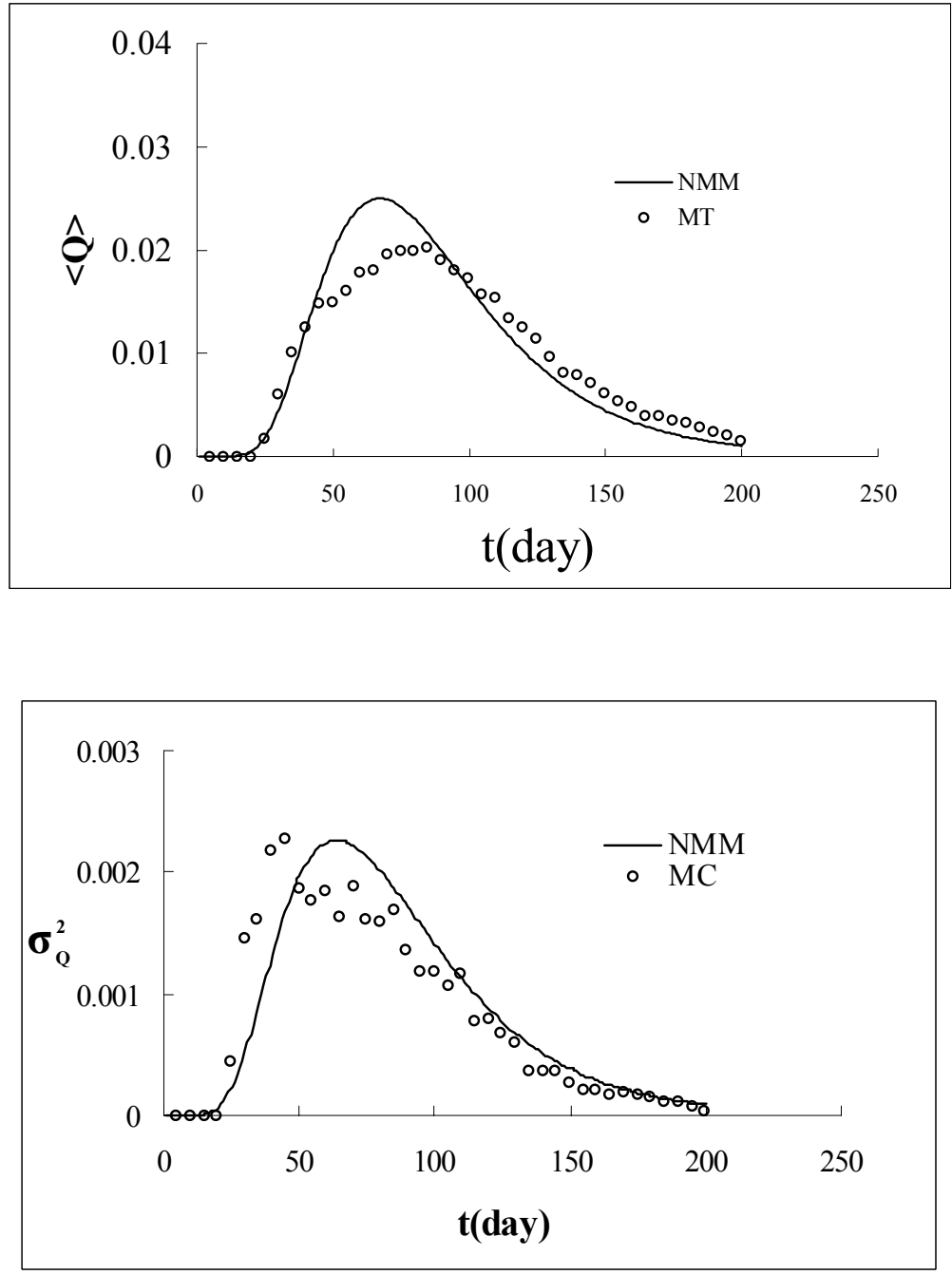

Figure 15 - Comparison of calculation results between Monte Carlo simulation and NMM method for case 10.

For the case study, we used a bimodal heterogeneous formation, which composed of two different materials, as an example to show how to apply the general theory to analyze the conductivity correlation in a specific case. Based on this analysis, we applied a numerical method of moment developed recently [Zhang et al., 2000; Wu et al., 2003] to investigate the influences of the two stochastic processes of heterogeneity on groundwater flow and transport. One specific case was chosen in this study, bimodal medium. We used the case study to investigate the influences of various uncertain factors on flow and transport predictions under different scenarios. We first investigate the influence of compositions of the two materials, $P(x)$, on flow and transport processes. It is shown from the study results that the variation of the $P(x)$ value has no effect on the mean head, 
but dramatically changes the head variance, mean and variance of solute flux. Based on this study, we propose 8 cases to study effects of other uncertainty parameters, which include the small-scale parameters, such as the variances and correlation lengths of log conductivity of the two materials, and large-scale parameters, such as the differences of mean $\log$ conductivity values of the two materials and the correlation length of the indicator function. The large-scale and small-scale heterogeneities will both significantly influence the flow and solute transport processes.

The NMM results are compared with those of the Monte Carlo simulation for the bi-model medium. The calculation results of the two methods are consistent with each other for small total variance of log-conductivity (less than 1.3 according to case 10), but will derivate with each other with the increase of the total variance.

\section{Acknowledgments}

This work was partially funded by DOE Yucca Mountain Project under contract between DOE and the University and Community College System of Nevada and Teaching and Research Award Program for Outstanding Young Teacher (TRAPOYT) of MOE, China.

\section{Appendix}

For $k=2, p_{2}(\mathbf{x})=1-p_{1}(\mathbf{x})$. Then we have

$$
\begin{aligned}
p_{11}(\mathbf{x}, \mathbf{s}) & =C_{I, 11}(\mathbf{x}, \mathbf{s})+p_{1}(\mathbf{x}) p_{1}(\mathbf{s}) \\
p_{12}(\mathbf{x}, \mathbf{s}) & =C_{I, 12}(\mathbf{x}, \mathbf{s})+p_{1}(\mathbf{x}) p_{2}(\mathbf{s}) \\
& =C_{I, 12}(\mathbf{x}, \mathbf{s})+p_{1}(\mathbf{x})-p_{1}(\mathbf{x}) p_{1}(\mathbf{s}) \\
p_{21}(\mathbf{x}, \mathbf{s}) & =C_{I, 21}(\mathbf{x}, \mathbf{s})+p_{2}(\mathbf{x}) p_{1}(\mathbf{s}) \\
& =C_{I, 21}(\mathbf{x}, \mathbf{s})+p_{1}(\mathbf{s})-p_{1}(\mathbf{x}) p_{1}(\mathbf{s}) \\
p_{22}(\mathbf{x}, \mathbf{s}) & =C_{I, 22}(\mathbf{x}, \mathbf{s})+p_{2}(\mathbf{x}) p_{2}(\mathbf{s}) \\
& =C_{I, 22}(\mathbf{x}, \mathbf{s})+1-p_{1}(\mathbf{x})-p_{1}(\mathbf{s})+p_{1}(\mathbf{x}) p_{1}(\mathbf{s})
\end{aligned}
$$

Since $p_{11}(\mathbf{x}, \mathbf{s})+p_{12}(\mathbf{x}, \mathbf{s})=p_{1}(\mathbf{x})$ and $p_{22}(\mathbf{x}, \mathbf{s})+p_{21}(\mathbf{x}, \mathbf{s})=1-p_{1}(\mathbf{x})$. Therefore, we can obtain equation (11). 


\section{REFERENCES}

[1] Care S.F. and Fogg G.E., Modeling spatial variabiliyy with one- and multi-dimensional continuous Markov chains, Math. Geology 29 (7) (1997), 891-918.

[2] Cushman J.H., The Physics of Fluids in Hierarchical Porous Media: Angstroms to Miles, Kluwer Academic, 1997.

[3] Cushman J.H, On measurement, scale, and scaling, Water Resour. Res. 22 (2) (1986), $129-134$.

[4] Dagan G., Stochastic theory of groundwater flow and transport: Pore to laboratory, laboratory to formation, and formation to regional scale, Water Resour. Res. 22 (9) (1986), 120S-134S.

[5] Dagan G., Flow and Transport in Porous Formations, Springer-Verlag, New York, 1989.

[6] Dagan G., Stochastic modeling of groundwater flow by unconditional and conditional probabilities, 2, The solute transport, Water Resour. Res. 18 (4) (1982), 835-848.

[7] Dagan G., Solute transport in heterogeneous porous formations, J. Fluid Mech. 145 (1984), $151-177$.

[8] Desbarats A.J., Numerical estimation of effective permeability in sand-shale formations, Water Resour. Res. 23 (2) (1987), 273-286.

[9] Desbarats A.J., Macrodispersion in sand-shale sequence, Water Resour. Res. 26 (1) (1991), $153-163$.

[10] Deutsch C.V and Journel A.G, GSLIB: Geostatistical Software Library and User's Guide, 2nd ed. Oxford University Press, New York, 369 pp, 1998.

[11] Gelhar L.W., Stochastic Subsurface Hydrology, Prentice-Hall, Englewood Cliffs, NJ, 1993.

[12] Hassan A.E., Cushman J.H. and Delleur J.W., A Monte Carlo assessment of Eulerian flow and transport peturbation models. Water Resour. Res. 34 (5) (1998), 1143-1163.

[13] Hoeksema R.J. and Kitanidis P.K., An application of the geostatistical approach to the inverse problem in two-dimensional groundwater modeling, Water Resour. Res. 20 (7) (1984), 10031020.

[14] Lu Z. and Zhang D., On stochastic modeling of flow in multimodal heterogeneous formations. Water Resour. Res. 38 (10) (2002), 10.1029/2001WR001026.

[15] Rubin Y. and Journel A.G., Simulation of non-Gaussian space random functions for modeling transport in groundwater, Water Resour. Res. 27 (7) (1991), 1711-1721.

[16] Rubin Y., Flow and transport in bimodal heterogeneous formations, Water Resour. Res. 31 (10) (1995), 2461-2468.

[17] Russo D., Zaidel J. and Laufer A., Numerical analysis of flow and transport in variably saturated bimodal heterogeneous porous media, Water Resour. Res. 37 (8) (2001), $2127-2141$. 
[18] Wheatcraft S.W. and Tyler S.W., An explanation of scale-dependent dispersivity in heterogeneous aquifers using concepts of fractal geometry, Water Resour. Res. 24 (4) (1998), 566-578.

[19] Winter C.L., Tartakovsky D.M. and Guadagnini A., Numerical solutions of moment equations for flow in heterogeneous composite aquifers, Water Resour Res. 38 (5) (2002), 10.1029/ 2001WR000222.

[20] Wu Jichun, Hu B. and Zhang D., Applications of nonstationary stochastic theories to solute transport in multi-scale geological media, Journal of Hydrology, 275 (3-4) (2003), 208-228.

[21] Zhang D., Stochastic Methods for Flow in Porous Media: Coping with Uncertainties, Academic Press, San Diego, Calif., 2002.

[22] Zhang D., Andricevic R. Sun A.Y., Hu B.X. and He G., Solute flux approach to transport through spatially nonstationary flow in porous media, Water Resour. Res. 36 (8) (2000), 2107-2120.

[23] Zhang D. and Winter C.L., Moment equation approach to single phase fluid flow in heterogeneous reservoirs, SPEJ Soc. Pet. Eng. J. 4 (2) (1999), 118-127. 